\title{
Progression of Cellular Adaptations in Medial Prefrontal and Orbitofrontal Cortex in Response to Repeated Amphetamine
}

\author{
Houman Homayoun and Bita Moghaddam \\ Department of Neuroscience, University of Pittsburgh, Pittsburgh, Pennsylvania 15260
}

\begin{abstract}
Recent theories on addiction implicate adaptive changes in prefrontal cortex (PFC) neurons in reinforcing and psychotomimetic properties of psychostimulants, yet little is known about how neuronal responses to these drugs change over time. Here we describe electrophysiological evidence for a progressive and sustained change in the response of PFC neurons to amphetamine during repeated exposure. In spontaneously behaving rats and in rats engaged in an instrumental responding task, we followed the activity of medial PFC (mPFC) and orbitofrontal cortex $(\mathrm{OFC})$ neurons during daily exposure to amphetamine and after a post-withdrawal challenge. Repeated amphetamine increased the number of responsive neurons and the magnitude of responses and modified spontaneous burst patterns. These changes were apparent after a few exposures to amphetamine, were amplified after withdrawal, and were region specific in that repeated amphetamine increasingly produced inhibitory responses in $\mathrm{MPFC}$ and excitatory responses in OFC. In behaviorally engaged animals, the gradual enhancement in MPFC inhibition and OFC overactivation correlated with a progressive impairment of instrumental responding. Furthermore, these changes were evident predominately in neurons that displayed phasic responses during task-related events. These rapid-onset and sustained cellular adaptations suggest that even limited exposure to psychostimulants may reduce the influence of mPFC neurons on behavior while at the same time exaggerating information encoded by OFC neurons.
\end{abstract}

Key words: psychostimulant; cortical plasticity; ensemble unit recording; freely moving rats; psychosis; addiction

\section{Introduction}

Adaptive responses of brain circuitry to drugs of abuse are thought to be critically involved in the development of addiction (Wise and Bozarth, 1987; Berke and Hyman, 2000; Robbins and Everitt, 2002; Nestler, 2004). For example, repeated use of psychostimulants such as cocaine or amphetamine produces increased behavioral responsiveness to these drugs (Robinson and Berridge, 1993). It has been hypothesized that the neuronal basis of these "learning" processes may underlie the detrimental effects of psychostimulant abuse, including persistent vulnerability to drug-seeking behaviors (Wolf, 2002; Jones and Bonci, 2005), and emergence of psychotic symptoms (Post and Kopanda, 1976; Bartlett et al., 1997; Castner and Goldman-Rakic, 2003). Although rodent studies have strongly implicated midbrain and striatal dopamine-rich regions in drug-induced plasticity (Everitt et al., 1999; Robinson and Berridge, 2002; Thomas and Malenka, 2003), clinical evidence suggest that the prefrontal cortex (PFC) may be a key substrate for adaptive responses to psychostimulants (Goldstein and Volkow, 2002; Kalivas et al., 2005). Specifically, functional imaging studies have consistently reported abnormal patterns of activity in orbital, medial, or dorsolateral PFC of individuals who abuse psychostimulants during baseline con-

Received Aug. 29, 2005; revised June 6, 2006; accepted June 6, 2006.

This work was supported by the National Institute of Mental Health and Pittsburgh Life Sciences Greenhouse. We thank Drs. Mark Jackson, Mark Stefani, and Charles Bradberry for helpful discussions and Alicia Defrancesco for technical assistance.

Correspondence should be addressed to Bita Maghaddam, Department of Neuroscience, University of Pittsburgh, A210 Langley Hall, Pittsburgh, PA 15260. E-mail: moghaddam@bns.pitt.edu.

DOI:10.1523/JNEUROSCI.0842-06.2006

Copyright $\odot 2006$ Society for Neuroscience $\quad$ 0270-6474/06/268025-15\$15.00/0 ditions or after challenge with drug or drug-associated cues (Volkow et al., 1992; Grant et al., 1996; London et al., 2000; Bolla et al., 2003). Consistent with these clinical findings, rodent studies show that repeated exposure to psychostimulants modifies dendritic morphology, membrane excitability, gene expression, and neurotransmitter release in subregions of PFC (Lu et al., 1997; Sorg et al., 1997; Crombag et al., 2004; Nestler, 2004; Robinson and Kolb, 2004; Dong et al., 2005). However, it remains a matter of debate whether PFC neurons are substrates for psychostimulant-induced plasticity or whether the long-term changes reported in cortical tissue are delayed consequences of changes in the traditional "reward circuits" involving midbrain and ventral striatum dopamine systems (Robbins and Everitt, 2002; Bonci et al., 2003; Kalivas et al., 2005).

To understand the dynamics of neuronal responses to repeated exposure to psychostimulants, we used an awake animal preparation that allowed us to assess progressive changes in spontaneous unit activity in the same animals in response to daily amphetamine injections and challenge injections of different doses after up to 3 weeks of withdrawal. Recordings were made in spontaneously behaving animals while they were in their home cage (experiment 1) and during performance of an instrumental responding task (experiment 2), a goal-directed behavior that engages PFC neurons (Mulder et al., 2003; Peters et al., 2005). This allowed us to determine whether any observed plasticity in the response of cortical neurons to repeated amphetamine was specific to periods of spontaneous behavior or generalized to periods of task engagement. Electrodes were implanted in the medial PFC (mPFC) and lateral orbitofrontal cortex (OFC) because these two cortical regions have abnormal activity in sub- 


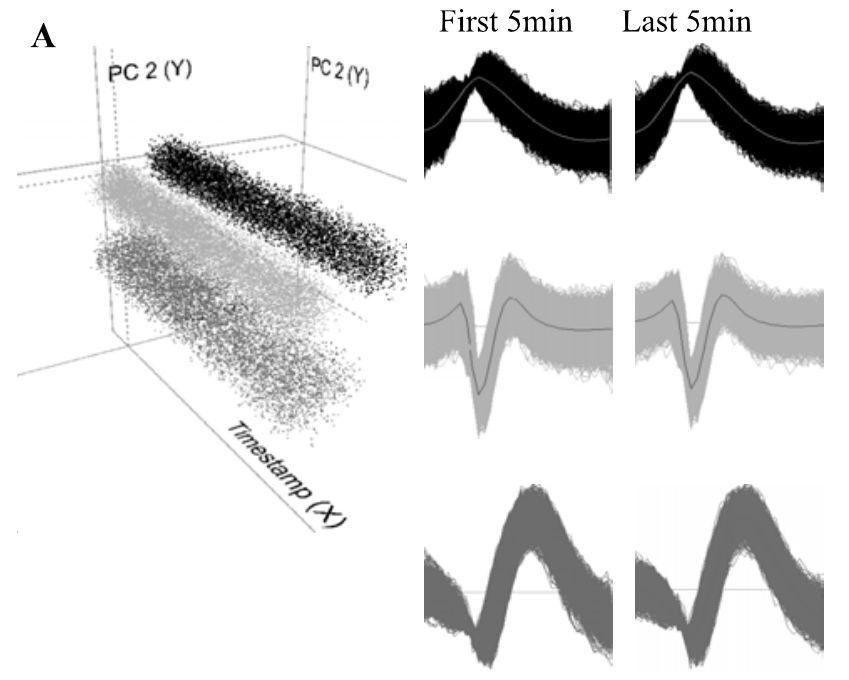

B

Experiment 1:

Amphetamine Treatment Schedule

\begin{tabular}{|c|c|c|c|c|}
\hline Dose: & $2 \mathrm{mg} / \mathrm{kg}$ & $2 \mathrm{mg} / \mathrm{kg}$ & $0.5 \mathrm{mg} / \mathrm{kg}$ & $2 \mathrm{mg} / \mathrm{kg}$ \\
\hline Days: & 12345 & 15 & 22 & 29 \\
\hline & & allenge1 & Challenge2 & Challen \\
\hline
\end{tabular}

\section{Experiment 2:}

Amphetamine Treatment Schedule

Dose: Veh $\frac{2 \mathrm{mg} / \mathrm{kg}}{\|\| \|} \quad 2 \mathrm{mg} / \mathrm{kg}$

Days: $0 \quad 12345$

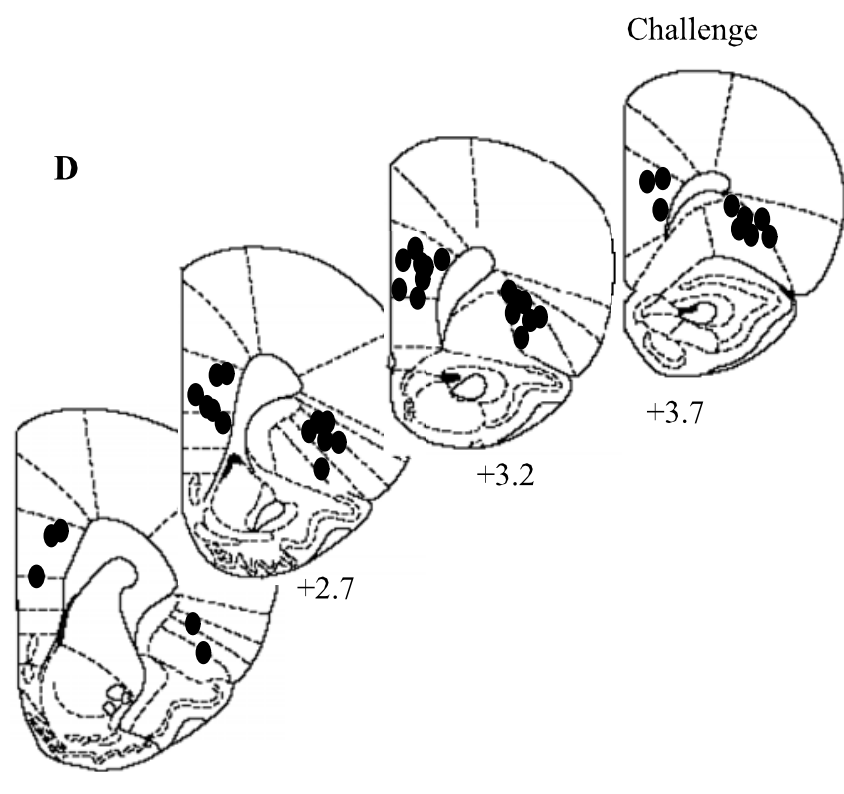

$+2.2$

Figure 1. Unit isolation, treatment schedule, and placement of electrodes. $A$, An example of single-unit isolation procedure in NeuroExplorer workspace. Three mPFC units have been separated as distinct clusters in three-dimensional space created based on the characteristics of spike waveforms using principal component ( $\mathrm{PC}$ ) analysis method. $\mathrm{PC} 1$ and $\mathrm{PC}(\mathrm{y}$ ( $z$ axis) are plotted against time ( $x$-axis) to examine the stability of each isolated unit throughout the stance abusers (London et al., 2000; Bolla et al., 2003; Ersche et al., 2005). During both behavioral states, we found that repeated amphetamine gradually produced larger and more sustained inhibitory responses in the $\mathrm{mPFC}$ and excitatory responses in the OFC.

\section{Materials and Methods \\ Subjects}

A total of 26 adult male Sprague Dawley rats weighing 340-420 g were used. Animals were individually housed on a $12 \mathrm{~h}$ light/dark cycle (lights on at 7:00 A.M.), and experiments were performed during the light phase. Rats in experiment 1 had access to food and water ad libitum, whereas those in experiment 2 were placed on a restricted food diet of $15 \mathrm{~g} / \mathrm{d} 1$ week before the start of training. Experimental procedures were approved by the University of Pittsburgh Institutional Animal Care and Use Committee and were conducted in accordance with the National Institutes of Health Guide for the Care and Use of Laboratory Animals.

\section{Electrophysiology}

Methodological details for recording and isolation of single units have been described previously (Homayoun et al., 2004; Jackson et al., 2004). Briefly, microelectrode arrays (NB Labs, Denison, TX) consisting of eight $50-\mu \mathrm{m}$-diameter Teflon-insulated, stainless steel wires were arranged in a $2 \times 4$ pattern measuring $\sim 0.25 \times 0.7 \mathrm{~mm}$. Electrodes were chronically implanted under halothane anesthesia in $\mathrm{mPFC}$ (target coordinates for the center of the array at anteroposterior, $+3.0 \mathrm{~mm}$; mediolateral, 0.7 $\mathrm{mm}$; and dorsoventral, $-3.5 \mathrm{~mm}$ ) or OFC (anteroposterior, $+3.0 \mathrm{~mm}$; mediolateral, $3.3 \mathrm{~mm}$; and dorsoventral, $-5.5 \mathrm{~mm}$ ) according to the atlas of Paxinos and Watson (1998). In experiment 1, recordings were made during spontaneous activity in a transparent standard cage with a modified open top. In experiment 2 , recordings were made while rats were engaged in an instrumental responding task in an operant conditioning box. In both experiments, animals were connected to a field-effect transistor head stage (NB Labs) using lightweight cabling that passed through a commutator and allowed the animal unrestricted movement during recording. Extracellular unit activity was recorded simultaneously from up to 16 channels using multiple channel amplifiers with $500 \times$ gain and $220-5900 \mathrm{~Hz}$ bandpass filters (Plexon, Dallas, TX). The amplified signal from each electrode was digitized ( $30 \mathrm{kHz}$ sampling rate) and saved on computer hard disk for off-line spike sorting. A minimum waveform amplitude threshold of 2 SDs higher than the noise amplitude was used for initial recording. Spike sorting was performed with Off-Line Sorter software (Plexon) using a combination of automatic and manual sorting techniques described previously (Homayoun et al., 2004). Only discrete clusters of units with stable characteristic waveforms and an absolute refractory period of at least $1.1 \mathrm{~ms}$ were accepted as single units. Waveforms of units isolated from the same electrode (principal components 1 and 2) were plotted against time to confirm stability of waveforms during each recording session (Fig. $1 A$ ). Whenever a shift in waveform was suspected, the corresponding unit was rejected. In addition, four different statistical measures were used to assess the quality of separation between identified clusters and the effects of incremental increases or decreases on the number of units isolated

$\leftarrow$

recording session. The template waveforms of each unit at the first (baseline) and last (after amphetamine injection) $5 \mathrm{~min}$ of recorded file are shown. The timestamp shows $20 \mathrm{~min}$ consisting of $10 \mathrm{~min}$ baseline and $10 \mathrm{~min}$ post-amphetamine recording. $\boldsymbol{B}$, Animals in experiment 1 received systemic injections of amphetamine $(2 \mathrm{mg} / \mathrm{kg})$ daily for $5 \mathrm{~d}$, followed by two challenges with the same dose on days 15 and 29 and a low-dose challenge $(0.5 \mathrm{mg} / \mathrm{kg})$ on day 22 . A control group received vehicle on days $1-5$ and challenge on day 15 . All injections were administered during spontaneous activity in a standard cage. C, Animals in experiment 2 were first trained on an FR1 instrumental responding task and were then administered vehicle (Veh) on day 0 and amphetamine ( $2 \mathrm{mg} / \mathrm{kg}$ ) daily for $5 \mathrm{~d}$ (days $1-5$ ), followed by a same-dose challenge on day 25 . Recordings were made during task performance on days $0,1,3,5$, and challenge. $\boldsymbol{D}$, Placement of electrode arrays $(0.7 \times 0.25 \mathrm{~mm})$ in $\mathrm{mPFC}$ (prelimbic-infralimbic) and OFC. Numbers under each coronal section indicate the distance anterior to bregma (Paxinos and Watson, 1998). 
Table 1. The number and baseline firing rates of neurons recorded during spontaneous activity (experiment 1)

\begin{tabular}{|c|c|c|c|c|c|}
\hline \multirow[b]{2}{*}{ Treatment } & \multirow[b]{2}{*}{ Session } & \multicolumn{2}{|c|}{$\mathrm{mPFC}$} & \multicolumn{2}{|l|}{$\mathrm{OFC}$} \\
\hline & & $n$ & Baseline (Hz) & $n$ & Baseline $(\mathrm{Hz})$ \\
\hline Amphetamine, $2 \mathrm{mg} / \mathrm{kg}$ & Day 1 & 188 & $3.35 \pm 0.32$ & 344 & $2.86 \pm 0.26$ \\
\hline Amphetamine, $2 \mathrm{mg} / \mathrm{kg}$ & Day 2 & 179 & $3.42 \pm 0.34$ & 310 & $3.13 \pm 0.33$ \\
\hline Amphetamine, $2 \mathrm{mg} / \mathrm{kg}$ & Day 3 & 184 & $3.73 \pm 0.27$ & 306 & $3.35 \pm 0.25$ \\
\hline Amphetamine, $2 \mathrm{mg} / \mathrm{kg}$ & Day 4 & 174 & $3.82 \pm 0.29$ & 291 & $3.16 \pm 0.27$ \\
\hline Amphetamine, $2 \mathrm{mg} / \mathrm{kg}$ & Day 5 & 182 & $3.61 \pm 0.28$ & 280 & $3.43 \pm 0.25$ \\
\hline Amphetamine, $2 \mathrm{mg} / \mathrm{kg}$ & Challenge 1 & 149 & $4.08 \pm 0.37$ & 173 & $3.22 \pm 0.28$ \\
\hline Amphetamine, $2 \mathrm{mg} / \mathrm{kg}$ & Challenge 3 & 69 & $3.95 \pm 0.34$ & 144 & $3.29 \pm 0.31$ \\
\hline Amphetamine, $0.5 \mathrm{mg} / \mathrm{kg}$ & Acute & 176 & $3.07 \pm 0.19$ & 97 & $2.83 \pm 0.24$ \\
\hline Amphetamine, $0.5 \mathrm{mg} / \mathrm{kg}$ & Challenge 2 & 139 & $3.58 \pm 0.22$ & 103 & $3.36 \pm 0.26$ \\
\hline Vehicle & Day 1 & 90 & $2.91 \pm 0.21$ & 79 & $2.68 \pm 0.23$ \\
\hline Vehicle & Day 5 & 80 & $2.74 \pm 0.22$ & 67 & $3.44 \pm 0.41$ \\
\hline Vehicle & Challenge 1 & 67 & $3.27 \pm 0.31$ & 70 & $3.27 \pm 0.42$ \\
\hline
\end{tabular}

Baseline (30 min) firing rates during different sessions were not significantly different in $\mathrm{mPFC}$ or OFC (one-way ANOVA, $p>0.05$ ).

ing task (see below). After reaching a stable performance level, animals received systemic injections of vehicle (day 0 ) or amphetamine $(2 \mathrm{mg} /$ $\mathrm{kg}$, days $1-5$, followed by a challenge dose on day 25) during performance of the operant task (Fig. 1C). After a $10 \mathrm{~min}$ session of instrumental responding (baseline), animals were removed from the operant box, injected, and returned to the box for another 40 min of behavior (after injection). Because animals showed progressive behavioral impairment (see Results), on injection day 3,5 , and challenge day, we placed them back in the operant box for a second $40 \mathrm{~min}$ session, $100 \mathrm{~min}$ after the injection. This late run allowed us to assess the duration of the effect of amphetamine on task performance during the repeated treatment. Note that this additional session was not applied during vehicle or day 1 amphetamine treatment because animals

from a channel. These included parametric $F$ statistics of multivariate ANOVA, the J3 and pseudo-F statistics, and the Davies-Bouldin (DB) validity index (Nicolelis et al., 2003). J3 is a measure of the ratio of between-cluster to within-cluster scatter, and DB is a measure of the ratio of the sum of within-cluster scatter to between cluster separation. Although none of these measures alone provides a clear-cut criterion for separating clusters, using them in conjunction with other criteria for cell isolation (above) improves the quality of unit isolation on a case-by-case basis (Nicolelis et al., 2003). A small subset of fast-firing neurons (mPFC, $2.5 \%$; OFC, $2.1 \%$ ) that showed characteristics of putative interneurons (spontaneous firing rate $>10 \mathrm{~Hz}$, tonic firing pattern in autocorrelogram) (McCormick et al., 1985; Jung et al., 1998) were excluded from analysis. Therefore, reported results are based on regular firing units (putative pyramidal neurons).

\section{Treatment}

Experiment 1. After 1 week of postsurgical recovery, animals were transferred from the housing room to the recording room and were gently handled and then connected to the head stage $3 \mathrm{~h}$ daily for $3 \mathrm{~d}$ to habituate to the recording environment. Thereafter, animals received daily systemic (intraperitoneal) injections of amphetamine $(2 \mathrm{mg} / \mathrm{kg}$, dissolved in saline) for $5 \mathrm{~d}$, followed by a $10 \mathrm{~d}$ "off drug" interval. Each animal then received three challenge injections of amphetamine as displayed in Figure $1 B$. Challenges consisted of two sessions of the same dose (2 mg/kg, days 15 and 29) and one session of a lower dose $(0.5$ $\mathrm{mg} / \mathrm{kg}$, day 22) of amphetamine. These different doses were used for challenge injections so that we could assess whether the effects of the same dose changes with repeated treatment and/or whether repeated treatment augments the effects of a lower dose. This amphetamine treatment schedule is consistent with previously established paradigms that induced significant behavioral sensitization (Badiani et al., 1995; Li and Wolf, 1999). A control group underwent a repeated vehicle (saline) treatment schedule (daily for $5 \mathrm{~d}$, followed by a challenge on day 15) to control for the effects of injection. All injections were administered $60 \mathrm{~min}$ after the animal's cage was placed in the recording chamber and electrodes were connected to the head stage. Each injection was preceded by $30 \mathrm{~min}$ of baseline and $150 \mathrm{~min}$ of postdrug recording. The neuronal responses on days $2-5$ and challenges 1 and 3 were compared with those after the same dose $(2 \mathrm{mg} / \mathrm{kg}$ ) on day 1 . Responses to challenge 2 (low dose) were compared with those after an acute injection of $0.5 \mathrm{mg} / \mathrm{kg}$ amphetamine in a separate group of animals. In experiment 1 , a total of 13 animals (three $\mathrm{mPFC}$, four OFC, and six both regions) underwent the repeated amphetamine treatment. An extra group of six (two mPFC, two OFC, and two both regions) was used for acute low-dose experiments. A separate control group of three rats (both $\mathrm{mPFC}$ and OFC) received repeated vehicle treatment.

Experiment 2. Initial habituation procedure was similar to experiment 1. In addition, animals were habituated to operant conditioning boxes 10 min daily for $3 \mathrm{~d}$. Starting on the second day of habituation, animals were connected to the head stage before being placed in the operant box. Animals were then trained on a fixed ratio 1 (FR1) instrumental respond- recovered responding behavior and obtained an above threshold number of food pellets (minimum of 60) during the initial 40 min postinjection run (see Results). A total of four rats (both mPFC and OFC implanted) were used in this experiment.

\section{Training procedure for instrumental responding}

An operant chamber (Coulbourn Instruments, Allentown, PA) equipped with an illuminating house light, three nose-poke modules, an illuminated food trough, and a food-delivery system was used. Nose-poke modules were placed on the wall opposite the food trough. Nose-poke modules could be illuminated by red light emitting diodes; the food trough could be illuminated by a white incandescent bulb. Head entries into the nose-poke modules or the pellet trough were detected by photosensors. A personal computer-based controller and data acquisition software were used (Graphic State Notation; Coulbourn Instruments). The chamber top was modified by installing a commutator that could be connected to recording cables. Event markers were used to synchronize the behavioral events with the electrophysiological spike trains. Animals were habituated for $10 \mathrm{~min}$ in each of 3 consecutive days, starting 5-7 d after electrode implantation surgery. During the first $5 \mathrm{~min}$, the house light was on and five food pellets (dextrose pellets, $45 \mathrm{mg}$; Bio-Serv, Frenchtown, NJ) were left in the food trough. During the second $5 \mathrm{~min}$, food pellets were intermittently dropped into the food trough in association with food-trough illumination. The pellet trough light remained on until the rat nose poked in the food trough, at which point a new cycle started with a food pellet dropping $20 \mathrm{~s}$ later.

Rats were then trained on an FR1 continuous reinforcement schedule to nose poke into an illuminated nose-poke module to receive a food reward. The house light was continuously illuminated for the duration of the session. After a $15 \mathrm{~s}$ period, the left nose-poke module was illuminated with red light. This cue light remained on until the first nose poke into the illuminated nose-poke module (instrumental response), at which time the red light would go off, a food pellet would be delivered into the food trough after a $100 \mathrm{~ms}$ delay (food delivery), and the food trough light was illuminated. The food-trough light remained on until the animal nose poked into the food trough (food consumption). A new cycle was initiated after a timeout period of $3 \mathrm{~s}$. The total number of delivered food pellets was recorded as the measure of instrumental responding. The food trough was inspected at the end of each session to ensure that animals were not engaged in operant responding without consuming reward. Drug treatment began 5-7 d after the start of training when rats reached the criterion of 120 correct responses per session for 3 consecutive days. Each training session consisted of a single run of 40 min. Each test session consisted of two runs of 10 and 40 min separated by $10 \mathrm{~min}$. Animals were injected between the two runs. Some amphetamine treatment sessions (days 3, 5, and challenge) included an additional $40 \mathrm{~min}$ run starting $100 \mathrm{~min}$ after injection (see above).

\section{Data analysis}

Electrophysiological data were analyzed using NeuroExplorer (Plexon) and Matlab (MathWorks, Natick, MA). Firing rate statistics were calcu- 


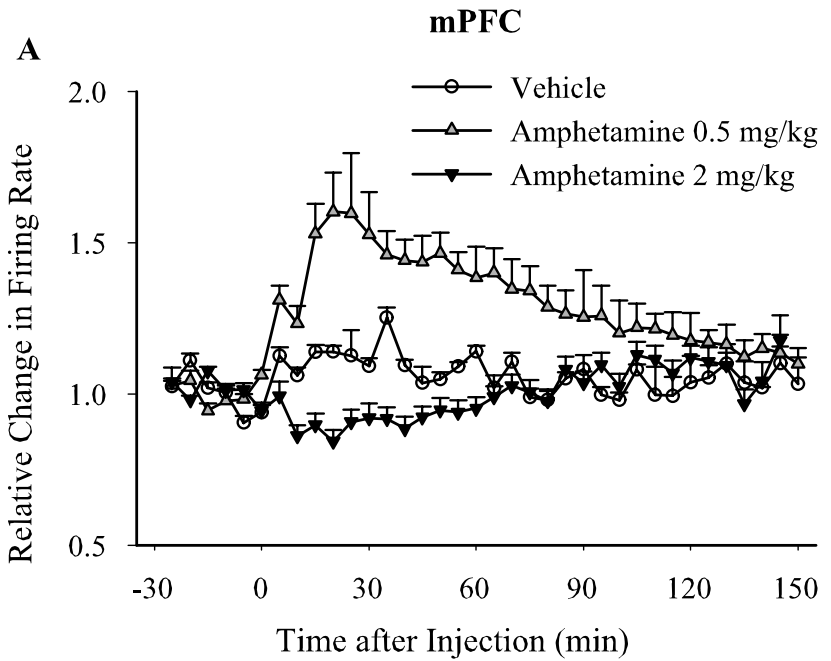

C
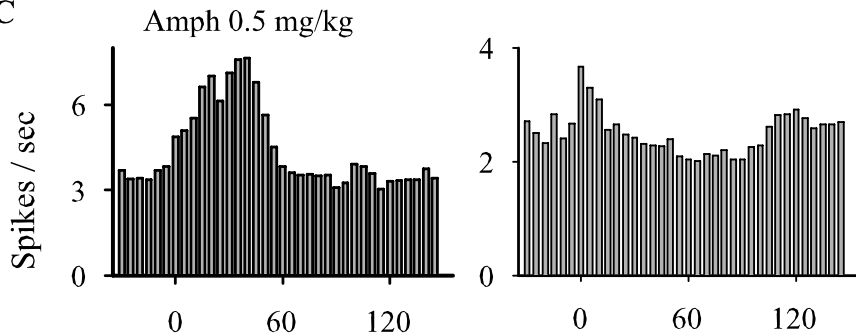

$\mathbf{E}$

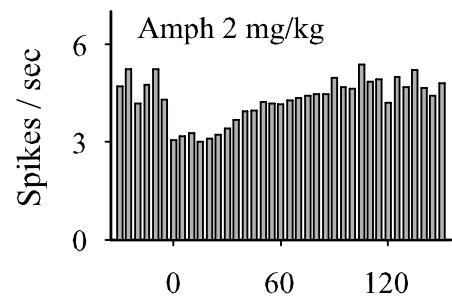

Time $(\min )$

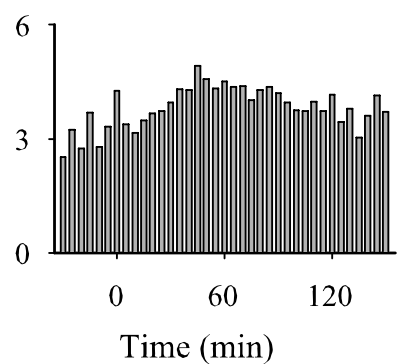

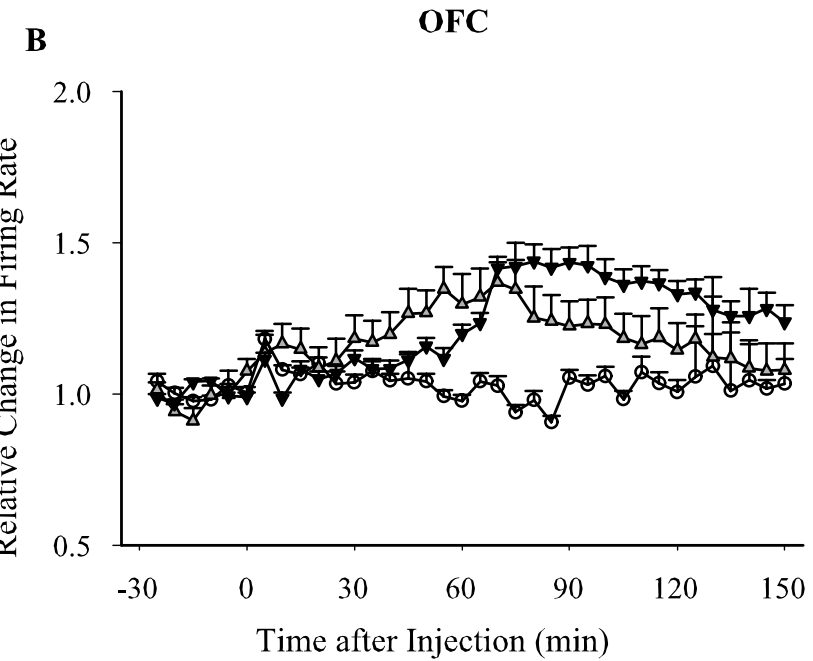

D
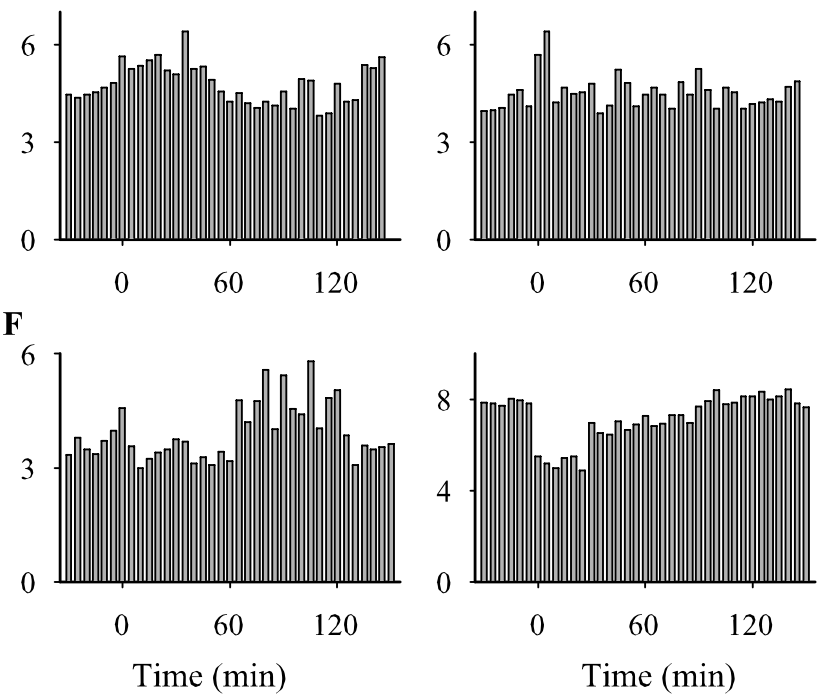

Figure 2. Dose-dependent effects of acute amphetamine. $\boldsymbol{A}, \boldsymbol{B}$, Average normalized firing rates of all $\mathrm{mPFC}(\boldsymbol{E})$ or $0 \mathrm{FC}(\boldsymbol{F})$ neurons treated with acute amphetamine or vehicle are compared. Two-way ANOVA with time as repeated measure revealed significant effects for both doses of amphetamine compared with vehicle in $\mathrm{mPFC}\left(0.5 \mathrm{mg} / \mathrm{kg}\right.$, treatment, $F_{(1,264)}=15.43, p<0.001$; time, $F_{(35,9240)}=9.17, p<0.001$; treatment $\times$ time interaction, $F_{(35,9240)}=17.15, p<0.001 ; 2 \mathrm{mg} / \mathrm{kg}$, treatment, $F_{(1,276)}=4.79, p<0.05 ;$ time, $F_{(35,9660)}=14.09, p<0.001 ;$ treatment $\times$ time interaction, $\left.F_{(35,9660)}=12.32, p<0.001\right)$ and $0 \mathrm{FC}\left(0.5 \mathrm{mg} / \mathrm{kg}\right.$, treatment, $F_{(1,174)}=3.74, p<0.05$; time, $F_{(35,6090)}=12.98, p<0.001 ;$ treatment $\times$ time interaction, $F_{(35,6090)}=11.13$, $p<0.001 ; 2 \mathrm{mg} / \mathrm{kg}$, treatment, $F_{(1,421)}=8.26, p<0.01$; time, $F_{(35,14735)}=34.70, p<0.001$; treatment $\times$ time interaction, $\left.F_{(35,14735)}=23.67, p<0.001\right)$. Time of injection is indicated as 0 . $\boldsymbol{C}-\boldsymbol{F}$, Sample firing rate histograms of individual single units ( $\mathrm{mPFC}, \boldsymbol{C}, \boldsymbol{E} ; 0 \mathrm{OFC}, \boldsymbol{D}, \boldsymbol{F})$ treated with acute amphetamine (Amph) $(0.5 \mathrm{mg} / \mathrm{kg}, \boldsymbol{C}, \boldsymbol{D} ; 2 \mathrm{mg} / \mathrm{kg}, \boldsymbol{E}, \boldsymbol{F})$. Each rate histogram (bin, $5 \mathrm{~min})$ shows the spontaneous firing rate of a single unit during $30 \mathrm{~min}$ of baseline and $150 \mathrm{~min}$ post-injection recording.

lated using normalized firing rate histograms with either 5 min bins (experiment 1) or $100 \mathrm{~s}$ bins (experiment 2). Different bin sizes were selected based on the length of each recording session. For each neuron, $99 \%$ confidence intervals of the baseline (30 $\mathrm{min}$ in experiment 1 and 10 min in experiment 2) firing rate were calculated, and postinjection bins that exceeded this range were marked as showing a significant effect. Two methods were used to assess the population dynamics of neuronal responses. First, the proportion of neurons that showed a significant response (increase or decrease) at each time bin was plotted against time to depict the temporal changes in the number of responsive versus nonresponsive units after each treatment. Second, neurons that showed sustained firing rate changes in response to amphetamine were identified and used to compare the effects of treatment on the firing rate magnitudes. For this analysis, a sustained response was defined as one starting with three consecutive $5 \mathrm{~min}$ bins (or six consecutive $100 \mathrm{~s}$ bins, experiment 2) exceeding the $99 \%$ confidence intervals of the baseline and terminating with three consecutive 5 min bins (or six consecutive $100 \mathrm{~s}$ bins) within the $99 \%$ confidence intervals of the baseline. Based on this criterion, neurons were classified as having one of the following sustained response types: unidirectional increase, unidirectional decrease, bidirectional response (both increase and decrease), and no sustained response. The distribution of response types was compared between groups using $\chi^{2}$ test. When this test indicated a significant between-group difference in the distribution of response types, we further assessed whether the proportion of a particular type of response (increase or decrease) was significantly altered by applying a $\chi^{2}$ retest after converting the response types into a binary variable (e.g., increase vs non-increase responses). This post hoc retest allowed us to distinguish whether a difference in the distribution of a given response is attributable to changes in that response per se or a result of altered distribution of other response types. Because the unidirectional responses constituted the majority of units in each amphetamine treatment session, we further compared the effects of treatment on normalized firing rates of neurons with sustained increase or decrease responses using two-way ANOVA with time as repeated measure and followed by Bonferroni's post hoc test. $p<0.05$ was considered the criteria for significance. 
mPFC

OFC

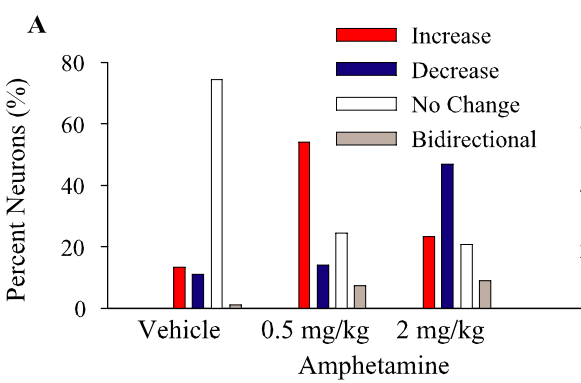

B

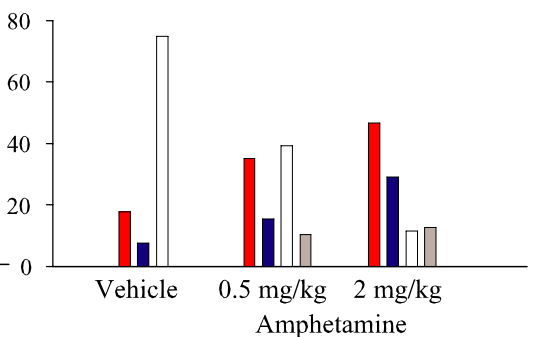

C

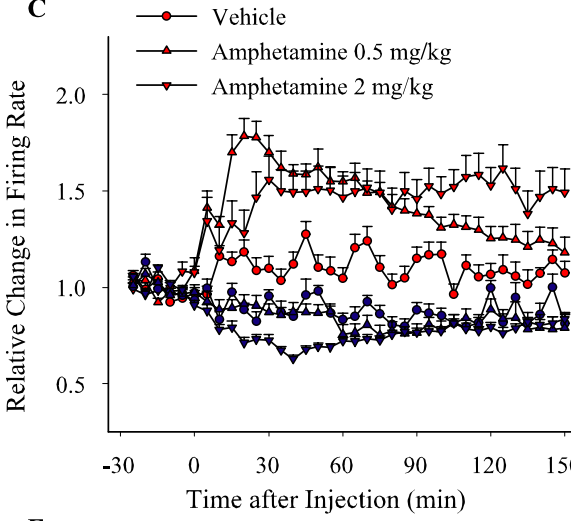

$\mathbf{E}$

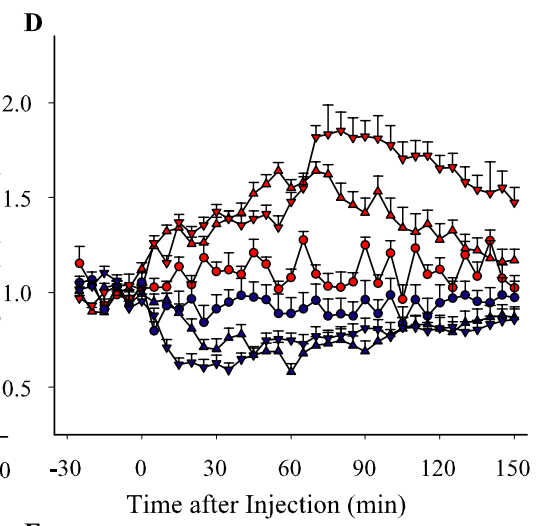
$\mathbf{F}$
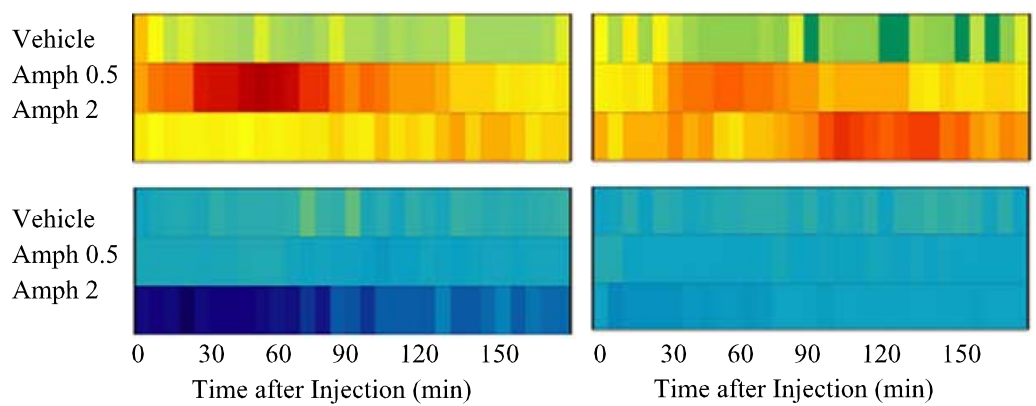

Figure 3. Types and dynamics of neuronal responses to acute amphetamine. $A, B$, Based on sustained changes in firing rates (see Materials and Methods), neuronal responses to treatment were classified as increase, decrease, no change, or bidirectional responses. The distribution of different response types induced by acute vehicle and low $(0.5 \mathrm{mg} / \mathrm{kg}$ ) and high ( $2 \mathrm{mg} / \mathrm{kg})$ doses of amphetamine in $\mathrm{MPFC}(\boldsymbol{A})$ and $\mathrm{OFC}(\boldsymbol{B})$ are compared. $\boldsymbol{C}, \boldsymbol{D}$, The average \pm SEM firing rates of neurons with sustained increase (red) or decrease (blue) responses. The average normalized firing rates of neurons with increase or decrease responses to each acute treatment have been separately depicted for mPFC (C) and OFC (D). Two-way ANOVA with time as repeated measure revealed that, compared with vehicle, both amphetamine doses caused significant excitatory effects in $\mathrm{mPFC}$ (increase responses, treatment, $F_{(2,148)}=5.28, p<0.005$; time, $F_{(35,5180)}=29.54, p<0.001$; treatment $\times$ time interaction, $F_{(70,5180)}=23.65$, $p<0.001$; post hoc test compared with vehicle, $p<0.05$ both doses) and OFC (treatment, $F_{(2,205)}=4.78, p<0.01$; time, $F_{(35,7175)}=21.50, p<0.001$; treatment $\times$ time interaction, $F_{(70,7175)}=8.06$; post hoc test, $p<0.05$ both doses $)$ and significant inhibitory effects in OFC (decrease responses, treatment, $F_{(2,118)}=3.41, p<0.05$; time, $F_{(35,4130)}=2.19, p<0.001$; treatment $\times$ time interaction, $F_{(70,665)}=3.23, p<0.001$; post hoc test, $p<0.05$ both doses). Only $2 \mathrm{mg} / \mathrm{kg}$ amphetamine caused a significant inhibitory effect in $\mathrm{mPFC}$ (treatment, $F_{(2,120)}=30.47, p<0.001$; time, $F_{(35,4200)}=6.02, p<0.001$; treatment $\times$ time interaction, $F_{(70,4200)}=10.27, p<0.001 ;$ post hoc test, $\left.0.5 \mathrm{mg} / \mathrm{kg}, p>0.05 ; 2 \mathrm{mg} / \mathrm{kg}, p<0.05\right) . \boldsymbol{E}, \boldsymbol{F}$, The temporal profile of population responses to acute treatments. Amph, Amphetamine. The density of momentary changes in firing rate is depicted by plotting against the time the proportion of $\mathrm{mPFC}(\boldsymbol{E})$ or OFC $(\boldsymbol{F})$ neurons that showed a significant firing rate increase (top panels) or decrease (bottom panels) at each post-injection time bin (5 min). Each horizontal color line is based on all neurons recorded on a given treatment day. Color codes for the density of increase and decrease responses (percentage of total) are shown in the color bar. Time of injection is indicated as 0 .

A reanalysis of sustained response types in experiment 1 was performed for early ( $\min 0-60)$ and late ( $\min 60-150)$ postinjection periods using the criteria of three consecutive $5 \mathrm{~min}$ bins (described above). A reanalysis of data in experiment 2 was performed using only periods of active postdrug instrumental responding (maximum inter-response interval of $20 \mathrm{~s}$ ) as window of analysis. This analysis allowed for comparison

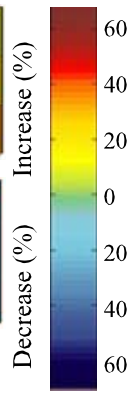

of firing rate during predrug and postdrug periods while the animal was engaged in a similar behavior (instrumental task). For each neuron, average firing rate during post-amphetamine active responding window was normalized to the firing rate during baseline active responding. The units displaying a minimum of $15 \%$ change in postdrug firing rate $(>115$ or $<85 \%)$ were considered to have a significant response. Because of the very low rates of behavioral responding on subsequent treatment days, this analysis was limited to day 1 amphetamine treatment.

The spike trains were also analyzed for measures of spontaneous bursting activity as an indicator of the temporal organization of spikes. We used a functional definition of bursting as periods in a spike train that have a higher discharge rate than do surrounding periods in the same spike train (Kaneoke and Vitek, 1996). Although PFC neurons in awake rats do not show periodic intrinsic bursting patterns similar to those observed in thalamic or dopaminergic neurons, their spiking organization is affected by afferent inputs in a way that leads to "burst periods" befitting the above definition (Homayoun et al., 2004). This type of bursting, which may be operationally described as periods of non-Poisson high-frequency firing, was detected by the Poisson surprise method of Legendy and Salcman (1985) as implemented in NeuroExplorer. This method detects bursts by locating consecutive interspike intervals (ISIs) that are less than half the mean ISI and testing whether these ISIs would be expected if the spike train were a Poisson process with the same mean firing rate. Thus, this analysis is not sensitive to changes in mean firing rate. The percentage of spikes in bursts during the postdrug period was compared between sessions using one-way ANOVA with Bonferroni's post hoc test. Burst analysis was performed only in experiment 1 because engagement in task performance during experiment 2 would not allow detection of "spontaneous" bursting.

Instrumental responding data were analyzed using one-way ANOVA with time as repeated measure for within-group analysis and two-way ANOVA with time as repeated measure and session as factor for between-group analyses. Pearson's correlation coefficient was used to assess correlation between the time course of behavioral responses and average neuronal firing for each treatment day. Furthermore, scatter plots depicting change in behavioral response rate versus change in neuronal firing were constructed. For this analysis, change in behavior was defined as change in the rate of correct responses per $100 \mathrm{~s}$ bins during the postdrug period compared with baseline. Change in firing activity was defined as average normalized firing rate of all units showing the predominant response type in each region or, alternatively, the average normalized firing of all units in each region. Linear regression was used for assessment of scatter plots. Phasic neuronal responses to significant behavioral events (cue onset, instrumental response, food delivery, and food consumption) were determined by plotting the perievent rasters and time histograms based on the first $10 \mathrm{~min}$ baseline of each session. Each perievent time histogram was constructed using $50 \mathrm{~ms}$ bins, smoothed by a Gaussian 
filter with bin width of 3 , and included a window of $\pm 2 \mathrm{~s}$ around the behavior of interest. Neuronal correlates of each behavioral event were analyzed during the following windows of analyses: -0.5 to $+0.5 \mathrm{~s}$ for cue onset, -1 to $+0.5 \mathrm{~s}$ for instrumental response, 0 to $+1 \mathrm{~s}$ for food delivery, and -0.5 to $+1 \mathrm{~s}$ for food consumption. Because the instrumental response and food delivery were separated by only $100 \mathrm{~ms}$, a phasic response that began by instrumental responding and was sustained after food delivery was considered instrumental response related, and, phasic responses, which exclusively started after food delivery, were considered food delivery related. A significant phasic response was defined if a minimum of four consecutive histogram bins (within window of analysis for each reference event) exceeded the $95 \%$ confidence intervals of the local baseline. Local baseline consisted of the window of -1.5 to -0.5 $\mathrm{s}$ before cue onset, during which animals showed minimum task-related behavior. For the units that displayed multiple perievent phasic responses, a retest was performed to determine whether there were true multiple phasic responses as opposed to a sustained change in firing in response to a single event. The baseline used for this retest was the $1.0 \mathrm{~s}$ period before the window of analysis for each significant event. Those phasic responses that met the significance criterion on both initial test and retest were accepted. Significant phasic responses were further visually examined to avoid erroneous classification. Subsequently, recorded neurons were classified as either "task encoder" (having at least one phasic event-related response) or "task nonencoder" (without any phasic response). To determine whether repeated amphetamine has an equal or preferential effect on firing rates of task encoder versus task non-encoder neurons, $\chi^{2}$ test was used to assess the distribution of firing rate responses to days 1,5 , and challenge injections of amphetamine within each subset.

The changes in the magnitude of the two most common types of phasic responses, consumption related and nose-poke related, were assessed using one-way ANOVA for within- and between-session comparisons. The magnitude of each phasic response was calculated according to a method adapted from Ghitza et al. (2006) using the ratio of $A /(A+B)$, with $A$ being the firing rate within the corresponding window of analysis $(-1$ to $+0.5 \mathrm{~s}$ for instrumental response and -0.5 to $+1 \mathrm{~s}$ for food consumption), and $B$ being the firing rate during the local baseline window ( -1.5 to $-0.5 \mathrm{~s}$ before cue onset; see above). Thus, a magnitude value between 0 and 0.5 indicates a phasic decrease, and a value between 0.5 and 1 indicates a phasic increase. The phasic magnitude was only calculated for neurons that showed a significant phasic response during predrug (baseline) task performance. The phasic response magnitude was then normalized by calculating the absolute value of its difference from 0.5 (Ghitza et al., 2006). This normalized ratio ranged between 0 (minimum change) and 0.5 (maximum change) and allowed for assessment of both excitatory and inhibitory phasic responses regardless of directionality. The change in response magnitude was calculated for these neurons by subtracting their baseline from postdrug normalized response magnitudes. The average of this value was used for withinsession analysis of drug effects on the magnitude of phasic responses. This analysis was performed for data obtained from days 1 and 3 of amphetamine treatment but could not be performed for day 5 and challenge data because of the severe behavioral impairment. Instead, a between-session analysis of the magnitude of phasic responses during baseline periods was performed using average magnitude of all significant phasic responses on a given session. For this analysis, phasic response magnitude was calculated as $A /(A+B), A$ being the peak change (excitatory or inhibitory) in firing rate within the corresponding window of analysis, and $B$ being the firing rate during the local precue baseline window (see above). The phasic responses were normalized as described above and compared between sessions. It should be noted that within-
mPFC
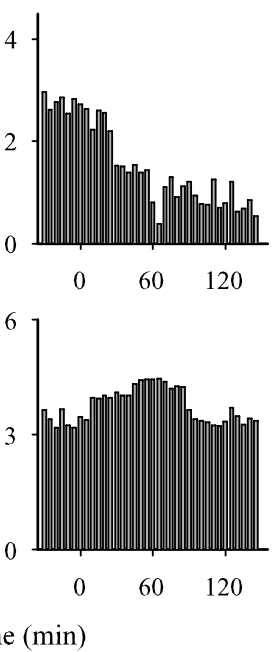

Time $(\mathrm{min})$
OFC

B
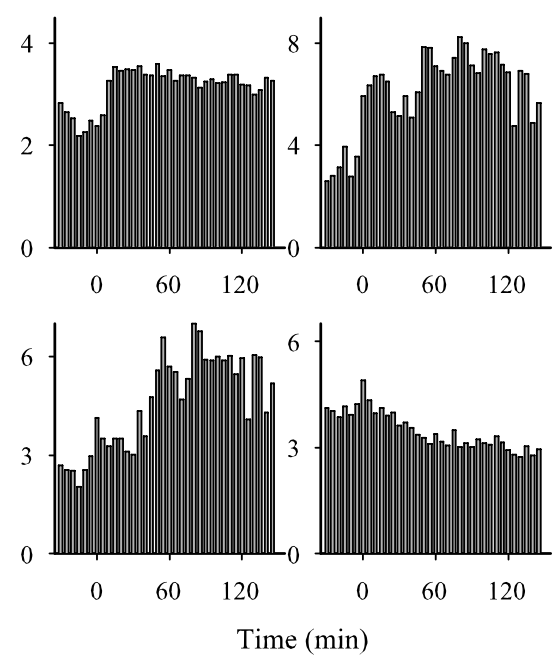

Figure 4. Sample firing rate histograms of individual single units ( $\mathrm{mPFC}, A ; \mathrm{OFC}, \boldsymbol{B})$ after amphetamine challenge $1(2 \mathrm{mg} / \mathrm{kg}$ ). Each rate histogram (bin, $5 \mathrm{~min}$ ) shows the spontaneous firing rate of a single unit during $30 \mathrm{~min}$ of baseline and $150 \mathrm{~min}$ post-injection recording. Time of injection is indicated as 0 .

session analysis of phasic responses focused on the drug effects on the encoding of task events by the same neurons, whereas the betweensession analysis examined whether previous treatment sessions affected phasic responses in the drug-free baseline epoch of a subsequent session.

\section{Histology}

Animals were anesthetized with chloral hydrate and intracardially perfused with saline, followed by $10 \%$ buffered Formalin. Fixed brains were cut at $200 \mu \mathrm{m}$ intervals, and sections were stained with cresyl violet to probe electrode placements. Under a light microscope, tips of recording electrodes were confirmed to be in the MPFC (prelimbic or dorsal infralimbic) or lateral OFC (Fig. 1D), as defined by the atlas of Paxinos and Watson (1998). Neurons recorded from electrodes that did not meet this criterion were excluded from analysis.

\section{Results}

\section{Experiment 1}

A total of 1677 and 2264 single units were recorded from $\mathrm{mPFC}$ and OFC, respectively. Neurons recorded from the same area during different sessions were treated as independent units, although fixed location of electrodes suggests that homogenous neuronal populations were recorded during various sessions. The baseline firing rates were not significantly different between sessions in either mPFC or OFC (Table 1).

\section{Effect of acute amphetamine}

Acute amphetamine treatment produced dose- and regionspecific patterns of activity in the mPFC and OFC. In the mPFC, amphetamine at $0.5 \mathrm{mg} / \mathrm{kg}$ increased and at $2 \mathrm{mg} / \mathrm{kg}$ decreased the average firing rate compared with vehicle (Fig. $2 A$ ). (Note that separate groups of amphetamine-naive animals were used for the data presented in Figure 2.) In the OFC, both doses of amphetamine increased the average firing rate (Fig. $2 \mathrm{~B}$ ). However, individual neurons in each group showed different patterns and magnitude of responses (Fig. 2C-F), suggesting that averaging the post-injection response may mask some of the treatment effects. Thus, we normalized firing rate histogram of each neuron and used two complementary types of analysis to follow the effect of amphetamine on (1) the pattern of response of individual neurons and (2) population dynamics. For the first set of analysis, individual neurons were first categorized based on their sustained 


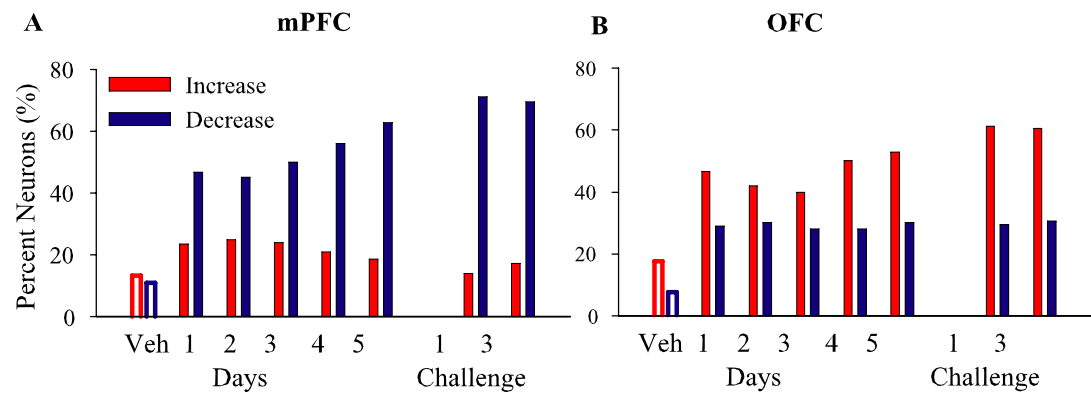

C

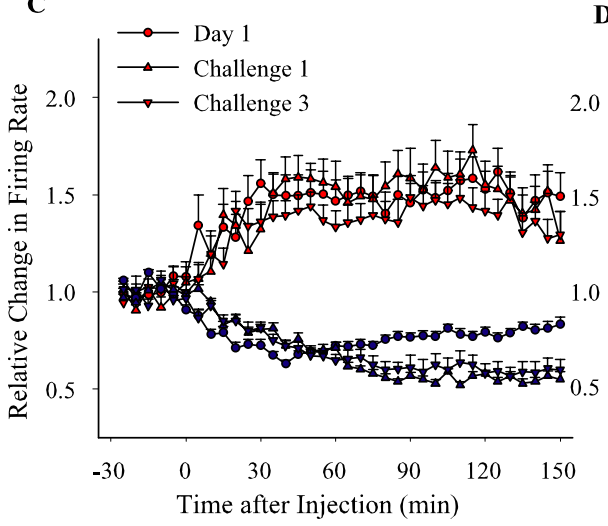

D

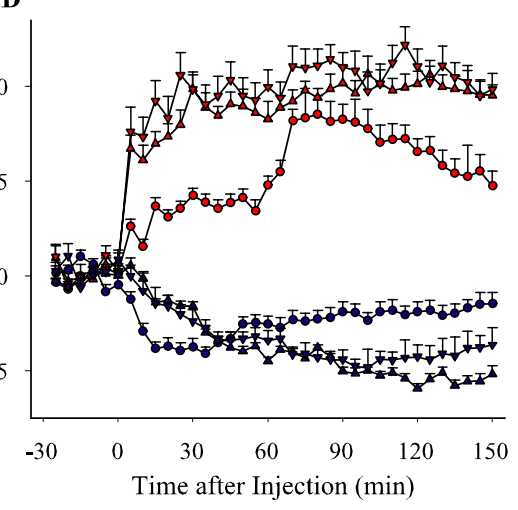

$\mathbf{E}$

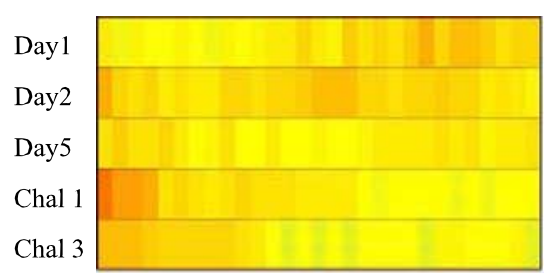

$\mathbf{F}$
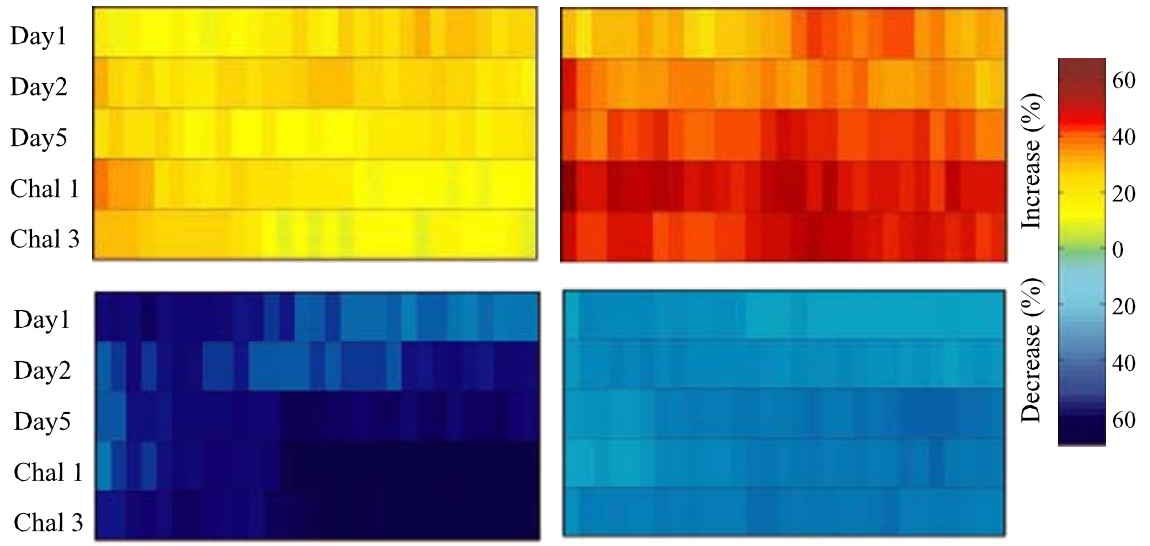

$\begin{array}{llllll}0 & 30 & 60 & 90 & 120 & 150\end{array}$

Time after Injection (min)

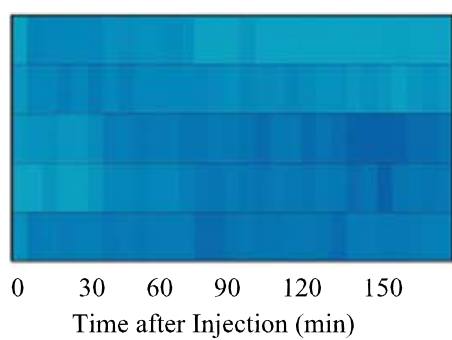

Figure 5. Effect of repeated amphetamine and same-dose challenges. $\boldsymbol{A}, \boldsymbol{B}$, The proportion of firing rate increase and decrease responses induced by the same dose of amphetamine $(2 \mathrm{mg} / \mathrm{kg}$ ) during repeated treatment (days $1-5)$ and subsequent challenges are compared in $\mathrm{MPFC}(\boldsymbol{A})$ and $\mathrm{OFC}(\boldsymbol{B}) . \chi^{2}$ test showed a significant change in the distribution of response types on day 5 ( $\left.\mathrm{mPFC}, \chi^{2}=19.47, p<0.001 ; 0 \mathrm{FC}, \chi^{2}=10.18, p<0.05\right)$, challenge 1 ( $\mathrm{mPFC}, \chi^{2}=36.75, p<0.001 ; 0 \mathrm{FC}$, $\chi^{2}=21.33, p<0.001$ ), and challenge 3 (mPFC, $\chi^{2}=18.18, p<0.001 ; \chi^{2}=16.77, p<0.001$ ) compared with day 1. For brevity, the proportion of the minority of neurons that showed no change or bidirectional response is not shown. Responses to day 1 vehicle (Veh) treatment are shown as open bars for comparison. $C, D$, The average \pm SEM firing rates of neurons with sustained increase (red) or decrease (blue) responses to repeated amphetamine in $\operatorname{mPFC}(\boldsymbol{C})$ and $0 F C(\boldsymbol{D})$. Both challenges 1 and 3 significantly enhanced the magnitude of firing rate excitations in $\mathrm{OFC}$ and inhibitions in both $\mathrm{MPFC}$ and $\mathrm{OFC}$ compared with day 1 (two-way ANOVAs with time as repeated measure, including day 1 and challenges 1 and $3 ; \mathrm{mPFC}$, increase responses, treatment, $F_{(2,74)}=2,9, p>0.05$; time, $F_{(35,2590)}=4.65, p<0.001$; treatment $\times$ timeinteraction, $F_{(70,2590)}=2.6, p<0.01$; post hoc comparison with day $1, p>0.05$; decrease responses, treatment, $F_{(2,239)}=5.9, p<0.005$; time, $F_{(35,8365)}=39.72$, $p<0.001$; treatment $\times$ time interaction, $F_{(70,8365)}=9.23, p<0.001 ; 0 \mathrm{FC}$, increase responses, treatment, $F_{(2,350)}=25.85$, $p<0.001$; time, $F_{(35,12250)}=34.50, p<0.001$; treatment $\times$ time interaction, $F_{(70,12250)}=16.74, p<0.001$; decrease responses, treatment, $F_{(2,188)}=3.24, p<0.05$; time, $F_{(35,6580)}=12.73, p<0.001$; treatment $\times$ time interaction, $F_{(35,6580)}=$ $5.16, p<0.001) . \boldsymbol{E}, \boldsymbol{F}$, The temporal profile of population responses to repeated amphetamine. The proportion of $\mathrm{MPFC}(\boldsymbol{E})$ or OFC $(\boldsymbol{F})$ neurons that showed a significant firing rate increase (top panels) or decrease (bottom panels) at each post-injection time bin $(5 \mathrm{~min})$ is plotted against time. In $\mathrm{mPFC}$, the peak density of neurons with inhibitory responses was observed after the first hour of amphetamine injection during challenges. In OFC, the peak density of neurons with excitatory responses occurred immediately after challenge amphetamine injection, whereas the peak density of neurons with inhibitory responses occurred after the first hour. All conventions are as in Figure 3. Chal 1, Challenge 1; Chal 3, challenge 3. firing rate responses to treatment. Thus, the post-injection response of individual neurons was classified as an increase, a decrease, no change, or bidirectional response (Fig. $3 A, B)$. The temporal profile and magnitude of firing rates of neurons in the increased and decreased firing response subgroups were then analyzed separately (Fig. $3 C, D)$. For the second set of analysis, the density of neuronal responses within the population was analyzed at each time bin. The proportion of neurons that showed a significant rate increase or decrease at any given time bin was plotted against time (Fig. $3 E, F)$. The majority of $\mathrm{mPFC}$ neurons responded to acute $0.5 \mathrm{mg} / \mathrm{kg}$ amphetamine by increasing their firing rate, whereas the predominant response to acute $2 \mathrm{mg} / \mathrm{kg}$ amphetamine was an inhibitory one (Fig. $3 A)$. In contrast, both doses caused predominantly excitatory responses in the OFC, with the higher dose producing more excitatory and inhibitory responses (Fig. $3 B$ ). In these groups, as well as in all of the subsequent groups, the proportion of neurons showing bidirectional changes in firing rate was low $(0-13 \%)$. In the $\mathrm{mPFC}$, the magnitude of firing rate increases was significantly enhanced by both doses of amphetamine compared with vehicle, whereas the magnitude of firing rate decreases was enhanced only by the $2 \mathrm{mg} / \mathrm{kg}$ dose (Fig. $3 C)$. In the OFC, the magnitude of both firing rate increases and decreases were enhanced by the low and high doses of amphetamine (Fig. 3D).

Analysis of regional population activity revealed that $0.5 \mathrm{mg} / \mathrm{kg}$ amphetamine increased the density of excitatory responses in both $\mathrm{mPFC}$ (Fig. 3E) and OFC (Fig. 3F), and $2 \mathrm{mg} / \mathrm{kg}$ amphetamine increased the density of inhibitory responses in $\mathrm{mPFC}$ and excitatory responses in OFC. Together, these results indicate that amphetamine produces dose- and region-specific effects on both the number of responsive units and their individual firing rates.

\section{Effect of repeated treatment and \\ same-dose challenges}

Neurons in both regions became more responsive to amphetamine $(2 \mathrm{mg} / \mathrm{kg})$ after repeated injections, as demonstrated by an increase in the relative number of responsive neurons. Furthermore, repeated amphetamine also modified the distribution of response types in a regionally selective manner. In the OFC, the relative number of neurons with an excitatory response to amphetamine increased, whereas in the mPFC, the proportion of inhibitory responses to amphetamine increased (Figs. $4 A, B$, sample individual firing rate histograms; $5 A, B$, 
population distribution). In contrast, there was no effect on the proportion of inhibitory neurons in the OFC or on excitatory neurons in the PFC $\left(\chi^{2}\right.$ retest after converting the response types into a binary variable, $p>0.05$; see Materials and Methods). The magnitudes of excitatory and inhibitory responses to same-dose amphetamine challenges were also affected in a regionally specific manner: in the $\mathrm{mPFC}$, only the magnitude of inhibitory responses was increased (Fig. 5C), whereas in the OFC, the magnitude of both excitatory and inhibitory responses was increased (Fig. 5D). In both regions, significant changes of similar nature and smaller magnitude were observed as early as day 2 (data not shown for brevity).

Changes in regional population responses to repeated amphetamine were also region specific (Fig. $5 E, F$ ). In the $\mathrm{mPFC}$, repeated amphetamine did not change the density of excitatory responses; however, an increase in the density of inhibitory responses was apparent after repeated injections (day 5) and became prominent on challenge days 1 and 3 (Fig. $5 E)$. In the OFC, there was an early increase in the density of excitatory responses accompanied with a more modest increase in the density of inhibitory responses on day 5 and challenge days 1 and 3 (Fig. $5 F$ ). Interestingly, changes in the density of responsive neurons (Fig. $5 E, F$ ) and the magnitude of their significant responses (Fig. $5 C, D$ ) followed distinct temporal dynamics in two regions. This distinction persisted when sustained firing response types were reanalyzed using two different windows of early (first hour) and late (after first hour) postdrug periods (supplemental Fig. 1, available at www.jneurosci.org as supplemental material). Amphetamine challenge caused an immediate excitatory peak in OFC neurons and a delayed inhibitory peak in mPFC.

\section{Effect of low-dose challenge}

In addition to potentiation of responses to the same dose of a drug, previous exposure influences the organism's response to a lower dose of that drug. Therefore, we challenged the amphetamine $(2 \mathrm{mg} / \mathrm{kg})$ preexposed animals with a low dose $(0.5 \mathrm{mg} / \mathrm{kg})$ of amphetamine and compared the results with the acute effects of the same low dose. In contrast to the predominantly excitatory responses induced by acute low-dose amphetamine in the mPFC (as described in Fig. 3A), low-dose challenge produced predominantly inhibitory responses, thus mimicking the acute effect of the higher dose of $2 \mathrm{mg} / \mathrm{kg}$ (Fig. 6A, inset). Moreover, the magnitude of sustained increase responses were lower, and decrease responses were higher than the effect of this low dose in amphetamine-naive animals (Fig. 6A). In the OFC, whereas acute low-dose amphetamine caused predominantly excitatory responses, the low-dose challenge increased the number of responsive cells, including both excitatory and inhibitory responses (Fig. $6 B$, inset), and potentiated the magnitude of both types of responses (Fig. 6B). Therefore, after repeated exposure to amChallenge2.
mPFC

OFC

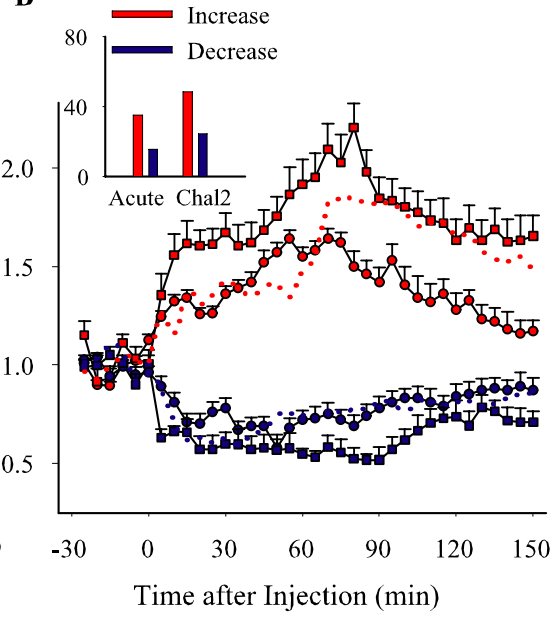

B

Time after Injection (min)

Figure 6. Effect of low-dose challenge. $\boldsymbol{A}, \boldsymbol{B}$, Insets, The proportion of firing rate increase or decrease responses to low-dose a of neurons. $A, B$ The average + SEM firing rates of neurons with sustained increase (red) or decrease (blue) responses to , $F_{(35,4200)}=4.33, p<0.001$; decrease responses, treatment, $F_{(1,99)}=7.04, p<0.01 ;$ time, $F_{(35,3465)}=2.61, p<0.05$ $F_{(1,38)}=3.84, p=0.05$; time, $F_{(35,1330)}=3.58, p<0.05$; treatment $\times$ time interaction, $\left.F_{(35,1330)}=2.58, p<0.05\right)$. Responses to acute $2 \mathrm{mg} / \mathrm{kg}$ amphetamine (day 1 ) is shown as a red dotted line for comparison. Time of injection is indicated as 0 . Chal2,

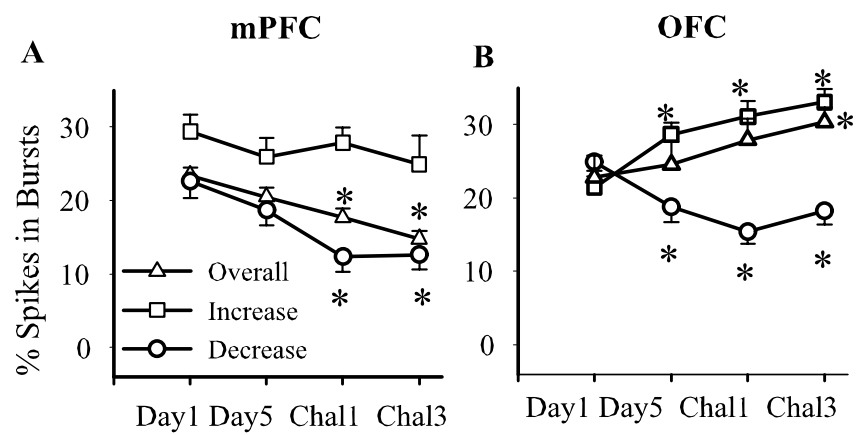

Figure 7. Effects on spontaneous bursting. Periods of spontaneous bursting were detected in each individual spike train by Poisson surprise method. The average \pm SEM percentage of spikes in bursts was calculated for each treatment day. Results are shown for all neurons recorded during a session (overall) and for the subsets of neurons with a sustained increase or decrease in firing rate. In $\mathrm{MPFC}(\boldsymbol{A})$, the amount of bursting (overall and neurons with decreased firing rate) was reduced on challenges 1 and 3 (one-way ANOVA; overall, $F_{(3,584)}=5.96, p<$ 0.01 ; neurons with a decrease firing response, $\left.F_{(3,349)}=7.15, p<0.001\right)$. In $0 \mathrm{FC}$, the overall bursting was significantly enhanced on challenge 3 (Chal3) but not on challenge 1 (Chal1) $\left(F_{(3,937)}=3.95, p<0.01\right)$. There was a significant enhancement of bursting in neurons with an increase firing response $\left(F_{(3,497)}=6.24, p<0.01\right)$ and a significant attenuation of bursting in neurons with a decrease firing response $\left(F_{(3,275)}=5.16, p<0.01\right)$ on day 5 and both challenge days compared with day $1 .{ }^{*} p<0.05$ compared with corresponding group on day 1 .

phetamine, effects of a lower dose of this drug on both mPFC and OFC neurons resemble the effects of a higher dose.

\section{Bursting}

Plasticity in neuronal response to amphetamine may also be expressed through non-rate codes such as clustering of spikes in 


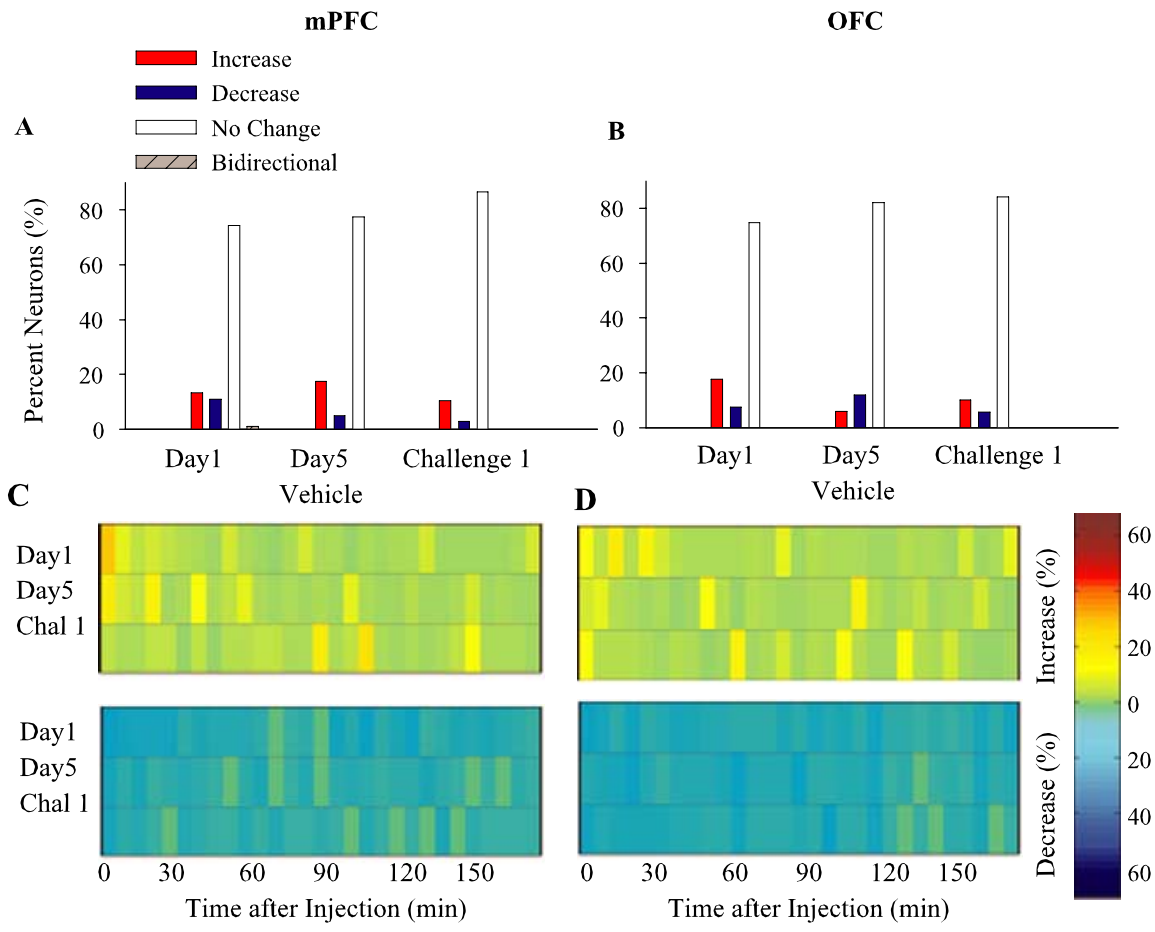

Figure 8. Effect of repeated vehicle treatment. $A, B$, The proportion of sustained firing rate responses induced by vehicle on day 1 , day 5 , and challenge 1 (day 15 ) in $\mathrm{mPFC}(\boldsymbol{A})$ and $0 \mathrm{FC}(\boldsymbol{B})$. The majority of neurons had no change in firing rate in response to different vehicle administrations. The distributions of response types were not different between sessions ( $p>0.05, \chi^{2}$ test). $C, D$, The temporal profile of population responses to repeated vehicle treatment. The density of momentary changes in firing rate is depicted by plotting against the time the proportion of $\mathrm{mPFC}(\boldsymbol{C})$ or $\mathrm{OFC}(\boldsymbol{D})$ neurons that showed a significant firing rate increase (top panels) or decrease (bottom panels) at each post-injection time bin ( $5 \mathrm{~min}$ ). Each horizontal color line is based on all neurons recorded on a given treatment day. Color codes for the density of increase and decrease responses (percentage of total) are shown in the color bar. Time of injection is indicated as 0 . Chal 1, Challenge 1.

Table 2. The number and baseline firing rates of neurons recorded during instrumental responding task (experiment 2)

\begin{tabular}{|c|c|c|c|c|c|}
\hline \multirow[b]{2}{*}{ Treatment } & \multirow[b]{2}{*}{ Session } & \multicolumn{2}{|c|}{$\mathrm{mPFC}$} & \multicolumn{2}{|c|}{ OFC } \\
\hline & & $n$ & Baseline $(\mathrm{Hz})$ & $n$ & Baseline $(\mathrm{Hz})$ \\
\hline Vehicle & Day 0 & 117 & $5.88 \pm 0.33$ & 96 & $6.30 \pm 0.33$ \\
\hline Amphetamine, $2 \mathrm{mg} / \mathrm{kg}$ & Day 1 & 104 & $6.21 \pm 0.34$ & 89 & $5.69 \pm 0.32$ \\
\hline Amphetamine, $2 \mathrm{mg} / \mathrm{kg}$ & Day 3 & 104 & $6.94 \pm 0.33$ & 90 & $6.21 \pm 0.34$ \\
\hline Amphetamine, $2 \mathrm{mg} / \mathrm{kg}$ & Day 5 & 112 & $6.51 \pm 0.31$ & 79 & $6.08 \pm 0.36$ \\
\hline Amphetamine, $2 \mathrm{mg} / \mathrm{kg}$ & Challenge & 86 & $6.62 \pm 0.37$ & 65 & $5.45 \pm 0.51$ \\
\hline
\end{tabular}

Although baseline firing rates during $10 \mathrm{~min}$ of instrumental responding were higher than spontaneous baseline rates recorded in experiment 1 , there were no significant between-session difference in mPFC or OFC (one-way ANOVA, $p>0.05$ ).

bursts. We determined the effects of treatment on an index of spontaneous bursting activity (percentage of spikes in bursts, detected by Poisson surprise method) in MPFC and OFC neurons. In the $\mathrm{MPFC}$, the overall bursting significantly decreased on challenges 1 and 3 compared with day 1 (Fig. 7A), an effect that was mainly driven by the subset of neurons with inhibitory firing rate responses. In the OFC, there was an increase in overall bursting in response to challenge 3 but not on challenge 1 (Fig. $7 B$ ). Additional analysis showed significant effects in different populations on day 5 and challenges 1 and 3 such that neurons with an excitatory firing rate response showed an increase in the bursting, whereas the population with an inhibitory response to amphetamine showed a decrease in the bursting (Fig. 7B).

\section{Effect of repeated vehicle treatment}

Repeated treatment did not cause any consistent change in neuronal responses in either $\mathrm{MPFC}$ or OFC. There were no significant between-session differences in the proportion of neurons with sustained firing rate responses (Fig. $8 A, B$ ) (mPFC, $\chi^{2}=8.02, p>0.05$; OFC, $\left.\chi^{2}=6.69, p>0.05\right)$. In addition, population activity during vehicle treatment sessions showed only transient and sporadic changes in firing rate (Fig. $8 C, D$ ). Moreover, repeated vehicle treatment had minimal effect on the percentage of spikes in bursts (comparison of days 1 and 5 and challenge with one-way ANOVA; mPFC, $F_{(2,234)}=0.27, p>0.05 ;$ OFC, $F_{(2,213)}=$ $1.23, p>0.05)$. Together, these data suggest that $\mathrm{mPFC}$ and OFC neuronal populations did not develop a sensitized response pattern to vehicle. Instead, vehicle-treated controls showed a gradual decrease in the number and magnitude of neuronal responses after repeated treatment, which may reflect a habituation to the mild stress of injection.

\section{Experiment 2}

This experiment was performed to assess the effects of repeated amphetamine on PFC neuronal firing in trained animals engaged in goal-directed behavior. A total of $523 \mathrm{mPFC}$ and 419 OFC neurons were recorded in 20 sessions from four rats during performance of an instrumental responding task. Baseline firing rates, determined during the initial $10 \mathrm{~min}$ of operant responding, were generally higher than baseline rates obtained during spontaneous activity in experiment 1 . However, baseline rates were not different between treatment sessions in the mPFC or the OFC (Table 2).

Vehicle treatment did not alter instrumental responding compared with baseline as assessed by repeated measures oneway ANOVA (Fig. 9A). However, repeated amphetamine led to a gradual disruption in instrumental responding. On day 1 , amphetamine produced a short-lasting disruption, with animals resuming a normal level of responding $30 \mathrm{~min}$ after injection. On day 3, there was a more sustained disruption, with animals reaching a normal level of responding during the late run (see Materials and Methods), $\sim 2 \mathrm{~h}$ after injection. By day 5 , animals showed minimal recovery in responding over $2 \mathrm{~h}$ after injection. In response to a challenge dose, there was no recovery during the late run (Fig. 9A). The baseline rate of instrumental responses was not different between treatment sessions.

To assess whether the effects of repeated amphetamine on neuronal firing rates observed in experiment 1 extends to behaviorally engaged animals, average normalized firing rates during each session were compared. Day 1 amphetamine caused a significant inhibition in $\mathrm{mPFC}$ (Fig. 9B) and excitation in OFC (Fig. $9 C$ ). Repeated amphetamine caused a progressively stronger inhibition on days 3,5 , and challenge in mPFC. In the OFC, the excitatory response to day 1 amphetamine was significantly potentiated on challenge day but not on days 3 or 5. Furthermore, during the late run session on day 3 , both $\mathrm{mPFC}$ inhibition and OFC excitation returned to baseline as behavioral responding 

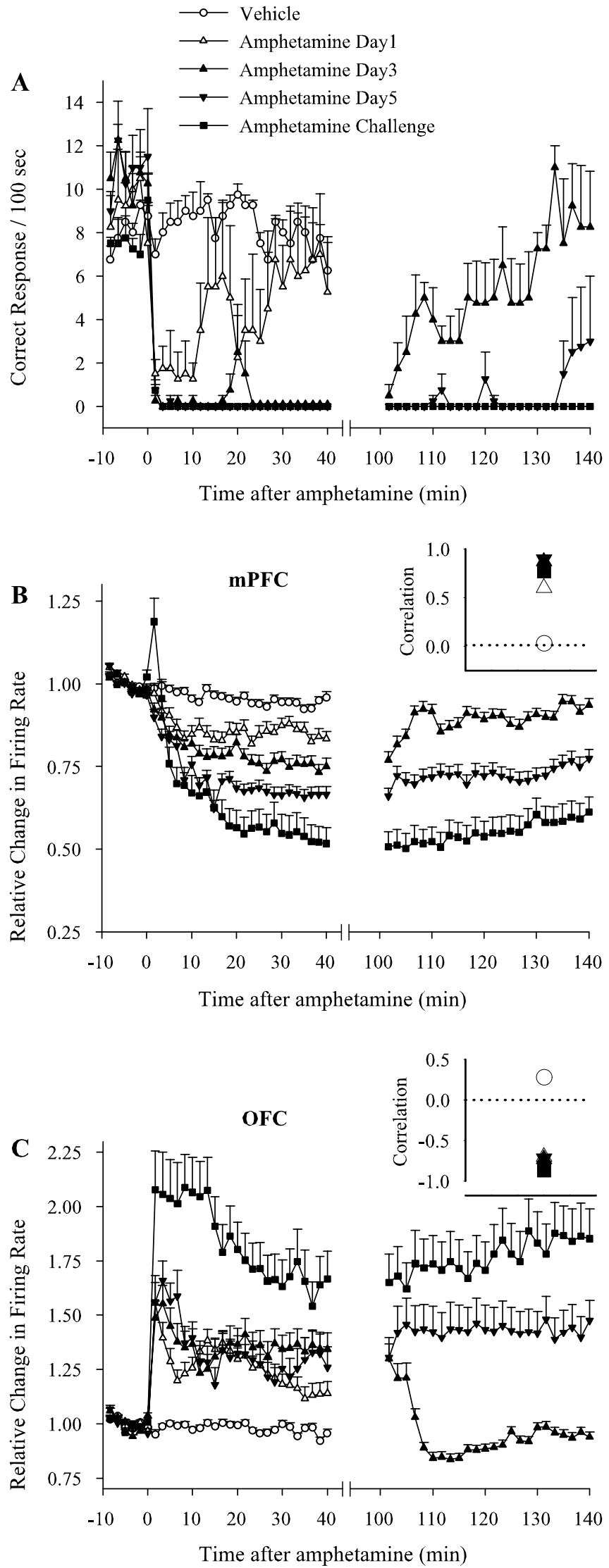

Figure 9. Behavioral and neuronal responses to amphetamine during instrumental responding task. $A$, Temporal profile of average \pm SEM instrumental responses during each treatment session. Each animal was run on a 10 min baseline instrumental responding task (FR1), followed by injection of vehicle or amphetamine $(2 \mathrm{mg} / \mathrm{kg})$ and $40 \mathrm{~min}$ of post-injection instrumental responding. Whenever drug treatment caused severe behavioral impairment

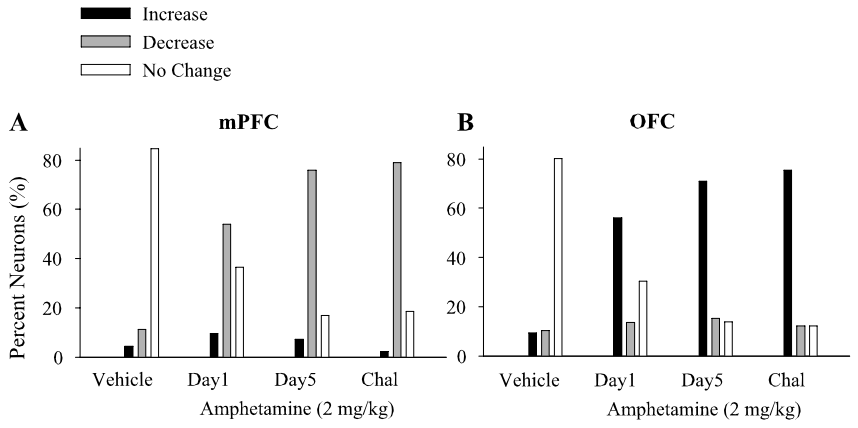

Figure 10. Types of neuronal responses to repeated amphetamine during instrumental responding task. $\boldsymbol{A}, \boldsymbol{B}$, Based on sustained changes in firing rate (see Materials and Methods), neuronal responses to treatment were divided into increase, decrease, or no change responses. The distribution of different response types induced by vehicle or days $1,3,5$, or challenge (Chal) of amphetamine are compared in $\mathrm{mPFC}(\boldsymbol{A})$ and $0 \mathrm{FC}(\boldsymbol{B}) \cdot \chi^{2}$ test showed a significant change in the distribution of responses to day 1 amphetamine compared with vehicle (mPFC, $\chi^{2}=$ $55.05, p<0.001 ; 0 \mathrm{FC}, \chi^{2}=52.52, p<0.05$ ). The proportions of decrease responses in $\mathrm{mPFC}$ and increase responses in $0 \mathrm{FC}$ were significantly enhanced on days 5 and challenge compared with day 1 (mPFC, day $5, \chi^{2}=12.24, p<0.01$; challenge day, $\chi^{2}=13.87, p<0.001 ; 0 \mathrm{FC}$, day $5, \chi^{2}=6.50, p<0.05$; challenge day, $\chi^{2}=7.56, p<0.05$ ).

was recovered. In contrast, mPFC neurons remained inhibited and OFC neurons excited as behavioral impairment persisted during late runs on days 5 and challenge. For all amphetamine treatment days, the time course of behavioral responding had a high positive correlation with $\mathrm{mPFC}$ firing (Fig. $9 B$, inset) and a high negative correlation with OFC firing (Fig. $9 C$, inset). These correlations were further verified by plotting the amphetamineinduced change in behavioral response rate of each rat against its average $\mathrm{mPFC}$ firing decreases (supplemental Fig. $2 \mathrm{~A}$, available at www.jneurosci.org as supplemental material) (linear regression, $r=0.71 ; p<0.001$ ) and OFC firing increases (supplemental Fig. $2 B$, available at www.jneurosci.org as supplemental material) $(r=0.53 ; p<0.05)$.

The effect of amphetamine on the proportion of neurons that

$\leftarrow$

(days 3,5 , and challenge), an additional 40 min run was allowed at 100-140 min after injection. Amphetamine caused a significant impairment in behavioral responding compared with vehicle (repeated measures two-way ANOVA; from minutes -10 to 40 ; treatment, $F_{(4,15)}=$ $17.61, p<0.001$; time, $F_{(29,435)}=38.31, p<0.001$; treatment $\times$ time interaction, $\left.F_{(116,435)}=4.37, p<0.001\right)$. The impairment was partial on day 1 but complete on days 3,5 , and challenge (significantly stronger than day 1 based on pairwise group comparisons with two-way ANOVA). In addition, behavioral responding was recovered during the late run on day 3 but not on days 5 or challenge (two-way ANOVA; from minutes -10 to 0 plus minutes 100 to $140 ; F_{(2,9)}=51.69, p<0.001$; time, $F_{(29,261)}=24.5, p<0.001$; treatment $\times$ time interaction, $\left.F_{(58,261)}=2.03, p<0.05\right)$. The $y$-axis shows the number of delivered food pellets during each $100 \mathrm{~s}$ bin. $\boldsymbol{B}, \boldsymbol{C}$, Average normalized firing rates of all $\mathrm{mPFC}(\boldsymbol{B})$ or $0 \mathrm{FC}(\boldsymbol{C})$ neurons recorded simultaneously with instrumental responding during each treatment day. Amphetamine treatment decreased the average firing rate in $\mathrm{MPFC}$ (two-way ANOVA with time as repeated measure; from minutes -10 to 40 ; treatment, $F_{(4,518)}=23.73, p<0.001$; time, $F_{(29,14993)}=$ $205.41, p<0.001$; treatment $\times$ time interaction, $\left.F_{(116,14993)}=19.80, p<0.001\right)$ and increased it in OFC (treatment, $F_{(4,413)}=19.55, p<0.001$; time, $F_{(29,11977)}=79.44, p<0.001$; treatment $\times$ time interaction, $\left.F_{(116,11977)}=13.72, p<0.001\right)$. The $\mathrm{mPFC}$ inhibition was significantly stronger on days 3,5 , and challenge compared with day 1 and on challenge day compared with day 5 (pairwise two-way ANOVAs). The $0 \mathrm{FC}$ excitation during post-injection run was not different between days 1,3 , or 5 but was stronger on challenge day compared with day 1 (pairwise two-way ANOVAs). In both regions, response to amphetamine challenge during the late run was significantly stronger than the response to day 3. $\boldsymbol{B}, \boldsymbol{C}$, Insets, Coefficients of correlations between average behavioral responding and average firing rates of $\mathrm{mPFC}(\boldsymbol{B}$, inset) or OFC ( $C$, inset) neurons during each treatment day. Instrumental responding was positively correlated with $\mathrm{mPFC}$ activity and negatively correlated with $\mathrm{OFC}$ activity during all amphetamine treatment days. Dotted line at 0 indicates no correlation. 

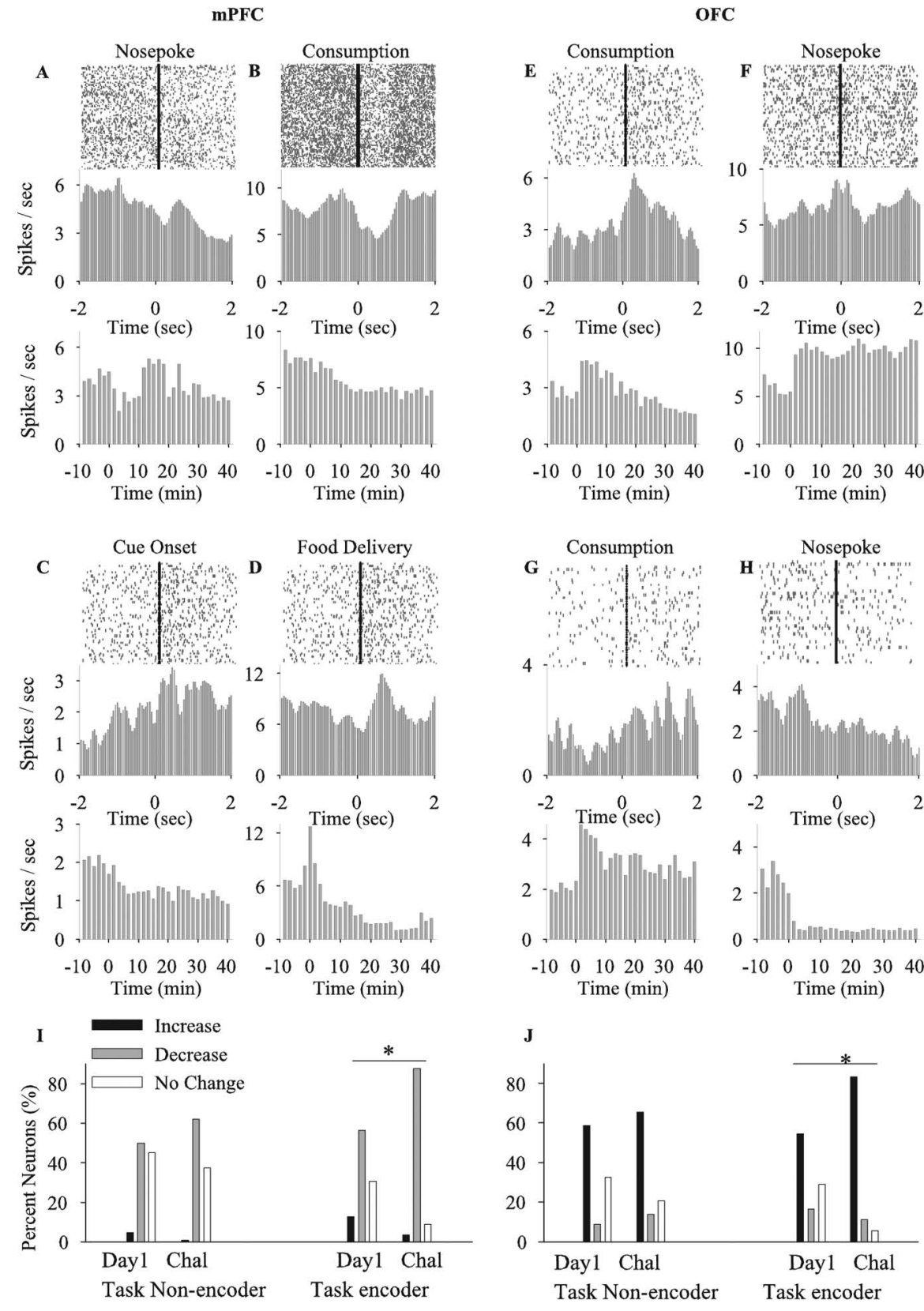

Figure 11. Effects of amphetamine on task encoder and task non-encoder neurons. $\boldsymbol{A}-\boldsymbol{H}$, Examples of mPFC $(\boldsymbol{A}-\boldsymbol{D})$ and $0 \mathrm{FC}$ $(\boldsymbol{E}-\boldsymbol{H})$ neurons exhibiting phasic firing rate responses to task-related events. Each section depicts the perievent raster (top panel) and time histogram (middle panel) of a single unit using 50 ms bins (smoothed) within a \pm 2 s perievent window. Events of interest, including cue onset, instrumental response, food delivery, and food consumption were used as reference to construct perievent plots. References are indicated above each raster plot. The bottom panel in each section depicts the firing rate histogram of the corresponding neuron in response to amphetamine using $100 \mathrm{~s}$ bins (non-smoothed) and a 50 min window (10 min task performance in baseline condition plus $40 \mathrm{~min}$ task performance after amphetamine). Amphetamine treatment: day 1 , units $\boldsymbol{A}, \boldsymbol{B}$; day 5 , units $\boldsymbol{E}, \boldsymbol{F}$; challenge, units $\boldsymbol{C}, \boldsymbol{D}$ and $\mathbf{G}, \boldsymbol{H}$. I, J, Comparison of the proportion of firing rate responses (increase, decrease, or no change) to days 1 and challenge of amphetamine within two subsets of task encoder and task non-encoder neurons in $\mathrm{mPFC}(\boldsymbol{I})$ and $\mathrm{OFC}(\boldsymbol{J})$. Within the task non-encoder subset, the proportion of responses to day 1 and challenge (Chal) of amphetamine was not different (mPFC, $\chi^{2}=2.05, p>0.05 ; 0 \mathrm{FC}, \chi^{2}=1.25, p>0.05$ ). In contrast, task encoder neurons showed a significantly higher proportion of $\mathrm{mPFC}$ inhibitory and OFC excitatory responses on challenge compared with day 1 ( $\mathrm{mPFC}, \chi^{2}=14.22, p=$ $\left.0.001 ; 0 \mathrm{FC}, \chi^{2}=9.28, p<0.05\right) .{ }^{*} p<0.05$ compared with day 1 .

increased or decreased their firing rates is shown in Figure 10. The majority of mPFC neurons decreased (Fig. 10A) and OFC neurons increased (Fig. $10 \mathrm{~B}$ ) their firing rates in response to day 1 amphetamine. The proportion of inhibitory responses in $\mathrm{mPFC}$ and excitatory responses in OFC was significantly enhanced during days 5 and challenge compared with day 1 . In addition, re- gional differences in measures of average firing activity and distribution of neuronal responses persisted when analysis was limited to postdrug periods in which animals were engaged in the same behavioral activity of nose-poke responding (supplemental Fig. 3, available at www.jneurosci.org as supplemental material). The similarity of these results with those obtained during the whole session and in the spontaneously active animals suggests that amphetamine effects on mPFC and OFC firing are unlikely to be an artifact of a particular behavioral state. However, we could not perform a similar comparison of firing rates during periods of task performance on subsequent treatment days as a result of severe behavioral impairment and, therefore, cannot rule out the possibility that expression of amphetamine-induced behaviors during these sessions may have contributed to changes in firing rates.

High correlation between amphetamine effects on neuronal activity and general task performance (Fig. 9) (supplemental Fig. 2, available at www.jneurosci.org as supplemental material) suggested that $\mathrm{MPFC}$ and OFC neurons may be substrates for detrimental effects of amphetamine on goaldirected behavior. To further investigate this notion, we analyzed the pattern of activity of neurons that displayed phasic responses during task-related events [cue onset, instrumental response, food delivery, and food consumption (examples shown in Fig. 11A$H)$ ]. We found that a high proportion of both $\mathrm{mPFC}$ and OFC neurons displayed task-related phasic responses (task encoders) (Table 3), particularly to food consumption and instrumental nose pokes. However, a subset of neurons in each region did not show any phasic responses and were classified as task non-encoders. The proportion of this subset of neurons was similar between different treatment sessions. We then asked whether repeated amphetamine has a similar or preferential effect on task encoder versus task non-encoder neurons. Within each subset, the proportion of firing rate responses to day 1 and challenge injections of amphetamine were compared (Fig. $11 \mathrm{I}, J$ ). In the task non-encoder subset, the proportion of responses on days 1 and challenge were similar in both regions. In the task encoder subset, a significantly higher proportion of neurons were inhibited in $\mathrm{MPFC}$ and excited in OFC on challenge day compared with day 1 . A similar pattern was observed on day 5 compared with day 1 (data not shown). Therefore, repeated amphetamine has increasingly disruptive influence on those $\mathrm{mPFC}$ and OFC neurons that had phasic responses to behavioral events.

The average magnitude of phasic responses to food consumption or nose pokes did not differ between sessions during baseline 
period, with the notable exception of a significant decrease in the magnitude of consumption-related responses in OFC on challenge day (supplemental Fig. 4A, B, available at www.jneurosci.org as supplemental material). However, amphetamine significantly attenuated the magnitude of consumption-related phasic responses (Fig. 12A-D, representative examples, $G$, $H$, within-session population analysis). This attenuation occurred for both phasic excitatory and inhibitory responses, suggesting an overall disruption in the neural representation of food. The magnitude of nose-poke-related phasic responses, on average, was not altered by amphetamine, although some individual neurons displayed significant change (Fig. $12 E, F$ ). An important limitation of this within-session analysis was that it only included periods of task performance during days 1 and 3 of amphetamine treatment. This inevitable sampling bias limits the interpretation of the results of this latter analysis, including the lack of difference in nose-poke-related phasic responses.

\section{Discussion}

Emergence of adaptive responses to repeated psychostimulant exposure may play a pivotal role in susceptibility to drug abuse and emergence of psychosis. We find that mPFC and OFC neurons are substrates for a rapidly emerging and enduring plasticity to amphetamine. The changes in the responses of $\mathrm{MPFC}$ and OFC neurons to amphetamine were expressed at the level of rate and pattern of spike firing, became more pronounced after 5 consecutive days of repeated treatment, and persisted for at least 3 weeks after withdrawal. These changes were apparent in spontaneously behaving animals and in animals engaged in a goal-directed behavior. In the latter group, the progressive effects of amphetamine were mostly observed in behaviorally engaged neurons, which showed phasic responses to task-related events.

The effects of amphetamine were different in the mPFC compared with the OFC. The initial exposure to amphetamine produced dose-dependent excitatory and inhibitory responses in both regions. Repeated amphetamine preferentially amplified inhibitory responses in the mPFC and excitatory responses in the OFC. Bursting also became less frequent in the mPFC but more prevalent in the OFC. This is significant because both the rate and pattern of single-unit firing in the PFC are critical for the maintenance of cognitive functions and reinforcement assessment that are served by these regions (Goldman-Rakic, 1995; Rolls et al., 1996; Cooper, 2002). Hence, the rate and burst data collectively indicate that repeated amphetamine produces a progressively greater functional inhibition of $\mathrm{MPFC}$ neurons and hyperactivation of OFC neurons.

Our results obtained in spontaneously behaving animals were confounded by the possibility that the behavioral state of the animal and/or environmental contingencies may affect neuronal responses to amphetamine. Thus, we performed another experiment assessing the effect of repeated amphetamine in animals that were engaged in an instrumental responding task and observed a similar pattern of plasticity in MPFC and OFC neurons. In particular, the region-specific effects of amphetamine were evident even when the analysis was limited to periods when animals were engaged in the same behavior, before and after amphetamine injection. These findings suggest that changes in the responsiveness of cortical neurons are not confined to a certain behavioral state and may represent a biological response to repeated amphetamine. Additional studies are needed to establish whether similar adaptations occur during contingent drugacquisition paradigms.

Behaviors served by $\mathrm{mPFC}$ (or dorsolateral PFC in primates) and OFC, such as executive control over goal-directed behavior or inhibition of impulsive behavior, are critically impaired in substance abusers (Grant et al., 2000; Bolla et al., 2003; Hester and Garavan, 2004; Kubler et al., 2005), leading to suggestions that $\mathrm{mPFC}$ - and OFC-dependent functions are a core component of addiction (Jentsch and Taylor, 1999; Volkow and Fowler, 2000; Bechara et al., 2001; Hyman and Malenka, 2001; Goldstein and Volkow, 2002). Although several studies have assessed the longterm effects of psychostimulants on PFC-dependent tasks (Schoenbaum et al., 2004; Castner et al., 2005; Fletcher et al., 2005), the present study provides direct evidence that the function of PFC neurons in behaving animals may be profoundly influenced by repeated amphetamine. We found that a progressive impairment in instrumental responding in a goal-directed behavior correlated with increasing inhibitory responses in the $\mathrm{mPFC}$. Furthermore, this cellular plasticity, i.e., progressive increase in inhibitory responses to the same dose of amphetamine, was primarily expressed in neurons that displayed a phasic response to task-related behaviors, suggesting that repeated exposure to psychostimulants may preferentially disrupt those mPFC ensembles that maintain goal-directed behavior. Of note, although lesion studies suggest that MPFC may not be necessary for instrumental responding (Ostlund and Balleine, 2005), electrophysiological studies show that MPFC neurons represent instrumental task events (Mulder et al., 2003; Peters et al., 2005; present data). For example, the attenuation and reestablishment of taskrelated representations in $\mathrm{mPFC}$ closely correlates with extinction and reacquisition of simple goal-directed responding (Peters et al., 2005).

The OFC neuronal ensembles encode the reinforcing values of stimuli and reward-predictive cues (Schoenbaum and Eichenbaum, 1995; Rolls et al., 1996; Tremblay and Schultz, 1999). Thus, the increasing excitatory responsiveness of OFC neurons, including the task-responsive neurons, to amphetamine may signify a higher salience assigned to the previously experienced drug. Our finding of a decline in the phasic response of OFC neurons to food in amphetamine-treated animals suggests that, as the drug becomes more salient, the salience of food diminishes. We hypothesize that the concomitant progression of inhibitory re- 

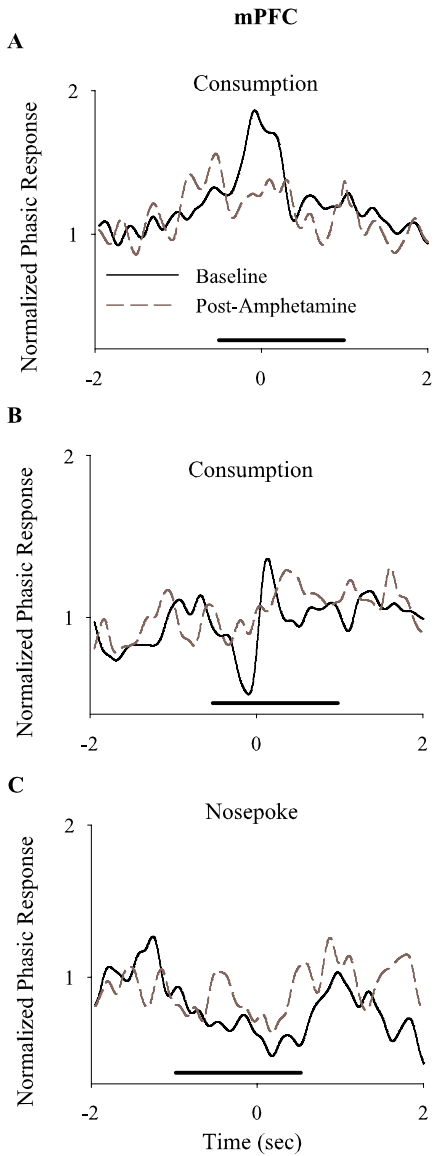

G

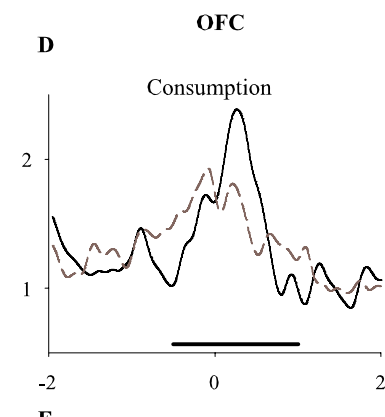

$\mathbf{E}$

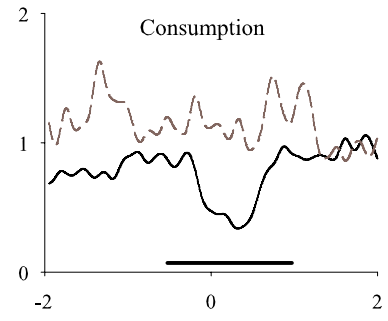

F

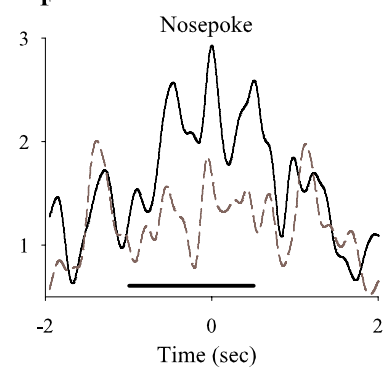

$\mathbf{H}$

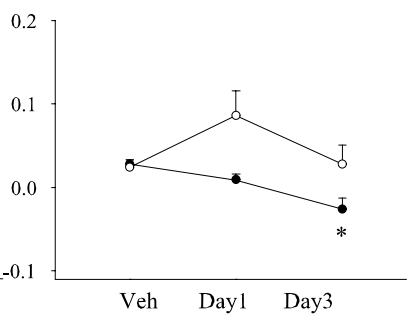

Figure 12. Effects of amphetamine on the magnitude of phasic task-related responses. $\boldsymbol{A}-\boldsymbol{F}$ Examples of mPFC $(\boldsymbol{A}-\boldsymbol{C})$ and OFC (D-F) individual phasic responses to task-related events ( $\boldsymbol{A}-\boldsymbol{D}$, food consumption; $\boldsymbol{E}, \boldsymbol{F}$, instrumental response) before (solid black line) and after (dashed gray line) amphetamine treatment ( $2 \mathrm{mg} / \mathrm{kg}$, day 3). Each section depicts the normalized perievent time histogram of a single unit using $50 \mathrm{~ms}$ bins (smoothed) within a $\pm 2 \mathrm{~s}$ perievent window. For each neuron, the histogram bins were normalized using the precue window ( -1.5 to $-0.5 \mathrm{~s}$ before cue) as described in Materials and Methods. The solid line depicts the window of analysis for each event. $A-D$, Examples of amphetamine attenuating the magnitude of phasic consumption-related responses $(\boldsymbol{A}, \boldsymbol{B}$, neurons with a phasic increase response; $\boldsymbol{C}$, a neuron with a phasic decrease-increase response; $\boldsymbol{D}$, a neuron with a phasic decrease response). $\boldsymbol{E}, \boldsymbol{F}$, Examples of amphetamine attenuating the phasic response to instrumental nose poke ( $\boldsymbol{E}$, a neuron with a phasic decrease response; $\boldsymbol{F}$, a neuron with a phasic increase response). $\boldsymbol{G}, \boldsymbol{H}$, Average within-session change in the magnitude of phasic responses in $\operatorname{mPFC}(\boldsymbol{G})$ and $\mathrm{OFC}(\boldsymbol{H})$. The normalized magnitude of each significant phasic response (regardless of directionality) was compared before and after amphetamine, and the differences were averaged per session. Amphetamine decreased the magnitude of consumption-related phasic responses in both regions (one-way ANOVA; $\mathrm{mPFC}, F_{(2,137)}=7.44, p<0.001 ; 0 \mathrm{FC}, F_{(2,134)}=10.13, p<0.001$ ) but did not alter the magnitude of nose-poke-related responses (mPFC, $F_{(2,49)}=2.63, p>0.05 ;$ OFC, $\left.F_{(2,91)}=1.93, p>0.05\right) .{ }^{*} p<0.05$ compared with vehicle.

sponses in the mPFC and excitatory responses in the OFC by amphetamine leads to reduced executive control of behavior at the same time as the motivational value of the drug is increasing. Simultaneous occurrence of these processes may contribute to development of addiction, which is associated with both failure in inhibition of impulsive behaviors and increased perceived salience of the drug.

Our findings are consistent with previous electrophysiological studies reporting inhibition of mPFC activity by amphetamine under anesthesia (Mora et al., 1976) and alteration in membrane excitability and evoked firing rate in vitro (Peterson et al., 2000; Dong et al., 2005; Nasif et al., 2005). Our observation of the rapid onset of plasticity at the level of PFC neuronal ensembles suggests that other measures of psychostimulant-induced plasticity with delayed onset (Wolf, 1998; Porrino and Lyons, 2000; Nestler, 2004; Robinson and Kolb, 2004) may be secondary to changes in cortical firing. These include changes in levels of c-Fos (Badiani et al., 1998), cAMP response element-binding protein (Garcia-Osta et al., 2004; Nestler, 2004), signaling machinery of NMDA receptors (Kalivas, 2001), and trafficking of AMPA receptors (Wolf, 2002; Boudreau and Wolf, 2005). In addition, recent evidence indicates that repeated dendritic branching is increased in the $\mathrm{mPFC}$ and decreased in the OFC after prolonged exposure to amphetamine (Robinson and Kolb, 1997; Crombag et al., 2004). Although these latter results have been generally interpreted as increased sensitivity of PFC to amphetamine (Robinson and Kolb, 2004), our findings suggest that they may represent a compensatory response to sustained inhibition of mPFC neurons and activation of OFC neurons by amphetamine.

One possible mechanism for the different effects of amphetamine in $\mathrm{MPFC}$ and OFC may be the degree in which amphetamine increases monoamine-mediated neurotransmission in these two regions. Amphetamine increases the synaptic availability of dopamine, norepinephrine, and serotonin (Moghaddam et al., 1990; Florin et al., 1994; Kuroki et al., 1996) by disrupting the function of monoamine transporters. This increase is sustained for 2-3 h, consistent with our observation of late significant responses. Differences in the density of monoamine transporters (Wang et al., 1995; Choi et al., 2000; Lewis et al., 2001; Logan et al., 2005) and magnitude of psychostimulant-induced monoamine release (Hedou et al., 2000; Bradberry and Rubino, 2004) have been reported in cortical subregions. The modulatory effects of monoamines in the PFC can be both excitatory and inhibitory: for example, dopamine exerts primarily an inhibitory influence in PFC neurons mediated by $\mathrm{D}_{1}$ receptors (TranthamDavidson et al., 2004), whereas serotonin primarily exerts an excitatory influence through $5-\mathrm{HT}_{2 \mathrm{~A}}$ receptors (Aghajanian and Marek, 1999). Therefore, proportionally larger amphetamineinduced serotonin release in the $\mathrm{OFC}$, and dopamine release in the $\mathrm{mPFC}$, may account for the predominantly excitatory versus inhibitory effects of amphetamine in these regions. Amphetamine has been shown to produce a more sustained increase in extracellular levels of dopamine compared with serotonin (Kuczenski and Segal, 1989), suggesting that a different temporal profile of increases in the extracellular levels of these monoamines may, in part, lead to the early excitations in the OFC versus delayed inhibitions in the mPFC. Although the magnitude of amphetamine-induced release of dopamine or serotonin may not increase after repeated exposure (Gartside et al., 1996), the initial surge in the release of these monoamines can elicit adaptive responses in postsynaptic targets by modifying glutamate neurotransmission such as increased trafficking of AMPA receptor subunits (Wolf, 2002) or expression of NMDA receptor-related scaffolding proteins (Szumlinski et al., 2004) so that the same level of increase in synaptic dopamine or serotonin can subsequently produce a greater modulatory influence on PFC neurons. Another possibility is that difference in afferent inputs, such as 
strong olfactory/gustatory inputs to OFC and segregated striatothalamic inputs to both regions (Uylings et al., 2003; Rolls, 2004), may underlie their distinct temporal pattern of responses to amphetamine.

In conclusion, we find that the response of mPFC neurons to amphetamine becomes progressively more inhibitory after repeated treatment, whereas the response of OFC neurons becomes more excitatory. These changes were predominately expressed by neurons that were engaged in task-related events, suggesting that repeated amphetamine preferentially modifies the function of cortical ensembles that subserve different aspects of goal-directed behavior. Given the evidence establishing that $\mathrm{mPFC}$ neurons sustain cognitive functions, such as reasoning and decision making, and OFC neurons encode the salient value of rewards, the present data may suggest that even limited exposure to amphetamine delivers a "double whammy" that may be critical for development of addiction or psychosis: it reduces the influence of $\mathrm{mPFC}$ on behavior at the same that it exaggerates the salient value of a rewarding experience.

\section{References}

Aghajanian GK, Marek GJ (1999) Serotonin, via 5-HT2A receptors, increases EPSCs in layer V pyramidal cells of prefrontal cortex by an asynchronous mode of glutamate release. Brain Res 825:161-171.

Badiani A, Morano M, Akil H, Robinson T (1995) Circulating adrenal hormones are not necessary for the development of sensitization to the psychomotor activating effects of amphetamine. Brain Res 673:13-24.

Badiani A, Oates M, Day H, Watson S, Akil H, Robinson T (1998) Amphetamine-induced behavior, dopamine release, and c-fos mRNA expression: modulation by environmental novelty. J Neurosci 18:10579-10593.

Bartlett E, Hallin A, Chapman B, Angrist B (1997) Selective sensitization to the psychosis-inducing effects of cocaine: a possible marker for addiction relapse vulnerability? Neuropsychopharmacology 16:77-82.

Bechara A, Dolan S, Denburg N, Hindes A, Anderson SW, Nathan PE (2001) Decision-making deficits, linked to a dysfunctional ventromedial prefrontal cortex, revealed in alcohol and stimulant abusers. Neuropsychologia 39:376-389.

Berke J, Hyman S (2000) Addiction, dopamine, and the molecular mechanisms of memory. Neuron 25:515-532.

Bolla K, Eldreth D, London E, Kiehl K, Mouratidis M, Contoreggi C, Matochik J, Kurianl V, Cadet J, Kimes A, Funderburk F, Ernst M (2003) Orbitofrontal cortex dysfunction in abstinent cocaine abusers performing a decision-making task. NeuroImage 19:1085-1094.

Bonci A, Bernardi G, Grillner P, Mercuri N (2003) The dopaminecontaining neuron: maestro or simple musician in the orchestra of addiction? Trends Pharmacol Sci 24:172-177.

Boudreau AC, Wolf ME (2005) Behavioral sensitization to cocaine is associated with increased AMPA receptor surface expression in the nucleus accumbens. J Neurosci 25:9144-9151.

Bradberry C, Rubino S (2004) Phasic alterations in dopamine and serotonin release in striatum and prefrontal cortex in response to cocaine predictive cues in behaving rhesus macaques. Neuropsychopharmacology 29:676-685.

Castner S, Goldman-Rakic P (2003) Amphetamine sensitization of hallucinatory-like behaviors is dependent on prefrontal cortex in nonhuman primates. Biol Psychiatry 54:105-110.

Castner S, Vosler P, Goldman-Rakic P (2005) Amphetamine sensitization impairs cognition and reduces dopamine turnover in primate prefrontal cortex. Biol Psychiatry 57:743-751.

Choi S, Hou C, Oya S, Mu M, Kung M, Siciliano M, Acton P, Kung H (2000) Selective in vitro and in vivo binding of $\left[{ }^{125} \mathrm{I}\right] \mathrm{ADAM}$ to serotonin transporters in rat brain. Synapse 38:403-412.

Cooper DC (2002) The significance of action potential bursting in the brain reward circuit. Neurochem Int 41:333-340.

Crombag H, Gorny G, Li Y, Kolb B, Robinson T (2004) Opposite effects of amphetamine self-administration experience on dendritic spines in the medial and orbital prefrontal cortex. Cereb Cortex 15:341-348.

Dong Y, Nasif F, Tsui J, Ju W, Cooper D, Hu X, Malenka R, White F (2005) Cocaine-induced plasticity of intrinsic membrane properties in prefron- tal cortex pyramidal neurons: adaptations in potassium currents. J Neurosci 25:936-940.

Ersche K, Fletcher P, Lewis S, Clark L, Stocks-Gee G, London M, Deakin J, Robbins T, Sahakian B (2005) Abnormal frontal activations related to decision making in current and former amphetamine- and opiatedependent individuals. Psychopharmacology 180:612-623.

Everitt BJ, Parkinson JA, Olmstead MC, Arroyo M, Robledo P, Robbins TW (1999) Associative processes in addiction and reward. The role of amygdala-ventral striatal subsystems. Ann NY Acad Sci 877:412-438.

Fletcher P, Tenn C, Rizos Z, Lovic V, Kapur S (2005) Sensitization to amphetamine, but not PCP, impairs attentional set shifting: reversal by a D1 receptor agonist injected into the medial prefrontal cortex. Psychopharmacology 183:190-200.

Florin S, Kuczenski R, Segal D (1994) Regional extracellular norepinephrine responses to amphetamine and cocaine and effects of clonidine pretreatment. Brain Res 654:53-62.

Garcia-Osta A, Del Rio J, Frechilla D (2004) Increased CRE-binding activity and tryptophan hydroxylase mRNA expression induced by 3,4methylenedioxymethamphetamine (MDMA, "ecstasy") in the rat frontal cortex but not in the hippocampus. Brain Res Mol Brain Res 26:181-187.

Gartside S, McQuade R, Sharp T (1996) Effects of repeated administration of 3,4-methylenedioxymethamphetamine on 5-hydroxytryptamine neuronal activity and release in the rat brain in vivo. J Pharmacol Exp Ther 279:277-283.

Ghitza UE, Prokopenko VF, West MO, Fabbricatore AT (2006) Higher magnitude accumbal phasic firing changes among core neurons exhibiting tonic firing increases during cocaine self-administration. Neuroscience 137:1075-1085.

Goldman-Rakic P (1995) Cellular basis of working memory. Neuron 14:477-485.

Goldstein R, Volkow N (2002) Drug addiction and its underlying neurobiological basis: neuroimaging evidence for the involvement of the frontal cortex. Am J Psychiatry 159:1642-1652.

Grant S, London E, Newlin D, Villemagne V, Liu X, Contoreggi C, Phillips R, Kimes A, Margolin A (1996) Activation of memory circuits during cueelicited cocaine craving. Proc Natl Acad Sci USA 93:12040-12045.

Grant S, Contoreggi C, London E (2000) Drug abusers show impaired performance in a laboratory test of decision making. Neuropsychologia $38: 1180-1187$.

Hedou G, Homberg J, Martin S, Wirth K, Feldon J, Heidbreder C (2000) Effect of amphetamine on extracellular acetylcholine and monoamine levels in subterritories of the rat medial prefrontal cortex. Eur J Pharmacol 390:127-136.

Hester R, Garavan H (2004) Executive dysfunction in cocaine addiction: evidence for discordant frontal, cingulate, and cerebellar activity. J Neurosci 24:11017-11022.

Homayoun H, Jackson ME, Moghaddam B (2004) Activation of metabotropic glutamate $2 / 3(\mathrm{mGlu} 2 / 3)$ receptors reverses the effects of NMDA receptor hypofunction on prefrontal cortex unit activity in awake rats. J Neurophysiol 93:1989-2001.

Hyman S, Malenka R (2001) Addiction and the brain: the neurobiology of compulsion and its persistence. Nat Rev Neurosci 2:695-703.

Jackson M, Homayoun H, Moghaddam B (2004) NMDA receptor hypofunction produces concomitant firing rate potentiation and burst activity reduction in the prefrontal cortex. Proc Natl Acad Sci USA 101:6391-6396.

Jentsch J, Taylor J (1999) Impulsivity resulting from frontostriatal dysfunction in drug abuse: implications for the control of behavior by rewardrelated stimuli. Psychopharmacology 146:373-390.

Jones S, Bonci A (2005) Synaptic plasticity and drug addiction. Curr Opin Pharmacol 5:20-25.

Jung M, Qin Y, McNaughton B, Barnes C (1998) Firing characteristics of deep layer neurons in prefrontal cortex in rats performing spatial working memory tasks. Cereb Cortex 8:437-450.

Kalivas P, Volkow N, Seamans J (2005) Unmanageable motivation in addiction: a pathology in prefrontal-accumbens glutamate transmission. Neuron 45:647-650.

Kalivas PW (2001) Drug addiction: to the cortex and beyond! Am J Psychiatry 158:349-350.

Kaneoke Y, Vitek JL (1996) Burst and oscillation as disparate neuronal properties. J Neurosci Methods 68:211-223.

Kubler A, Murphy K, Garavan H (2005) Cocaine dependence and attention 
switching within and between verbal and visuospatial working memory. Eur J Neurosci 21:1984-1992.

Kuczenski R, Segal D (1989) Concomitant characterization of behavioral and striatal neurotransmitter response to amphetamine using in vivo microdialysis. J Neurosci 9:2051-2065.

Kuroki T, Ichikawa J, Dai J, Meltzer H (1996) $R(+)-8-O H-D P A T$, a 5-HT1A receptor agonist, inhibits amphetamine-induced serotonin and dopamine release in rat medial prefrontal cortex. Brain Res 743:357-361.

Legendy CR, Salcman M (1985) Bursts and recurrences of bursts in the spike trains of spontaneously active striate cortex neurons. J Neurophysiol 53:926-939.

Lewis D, Melchitzky D, Sesack S, Whitehead R, Auh S, Sampson A (2001) Dopamine transporter immunoreactivity in monkey cerebral cortex: regional, laminar, and ultrastructural localization. J Comp Neurol 432:119-136.

Li Y, Wolf M (1999) Can the "state-dependency" hypothesis explain prevention of amphetamine sensitization in rats by NMDA receptor antagonists? Psychopharmacology 141:351-361.

Logan J, Ding Y, Lin K, Pareto D, Fowler J, Biegon A (2005) Modeling and analysis of PET studies with norepinephrine transporter ligands: the search for a reference region. Nucl Med Biol 32:531-542.

London E, Ernst M, Grant S, Bonson K, Weinstein A (2000) Orbitofrontal cortex and human drug abuse: functional imaging. Cereb Cortex 10:334-342.

Lu W, Chen H, Xue C, Wolf M (1997) Repeated amphetamine administration alters the expression of mRNA for AMPA receptor subunits in rat nucleus accumbens and prefrontal cortex. Synapse 26:269-280.

McCormick DA, Connors BW, Lighthall JW, Prince DA (1985) Comparative electrophysiology of pyramidal and sparsely spiny stellate neurons of the neocortex. J Neurophysiol 54:782-806.

Moghaddam B, Roth RH, Bunney BS (1990) Characterization of dopamine release in the rat medial prefrontal cortex as assessed by in vivo microdialysis: comparison to the striatum. Neuroscience 36:669-676.

Mora F, Sweeney K, Rolls E, Sanguinetti A (1976) Spontaneous firing rate of neurones in the prefrontal cortex of the rat: evidence for a dopaminergic inhibition. Brain Res 116:516-522.

Mulder A, Nordquist R, Orgut O, Pennartz C (2003) Learning-related changes in response patterns of prefrontal neurons during instrumental conditioning. Behav Brain Res 146:77-88.

Nasif F, Sidiropoulou K, Hu X, White F (2005) Repeated cocaine administration increases membrane excitability of pyramidal neurons in the rat medial prefrontal cortex. J Pharmacol Exp Ther 312:1305-1313.

Nestler E (2004) Molecular mechanisms of drug addiction. Neuropharmacol 47 [Suppl 1]:24-32.

Nicolelis MAL, Dimitrov D, Carmena JM, Crist R, Lehew G, Kralik JD, Wise SP (2003) Chronic, multisite, multielectrode recordings in macaque monkeys. Proc Natl Acad Sci USA 100:11041-11046.

Ostlund SB, Balleine BW (2005) Lesions of medial prefrontal cortex disrupt the acquisition but not the expression of goal-directed behavior. J Neurosci 25:7763-7770.

Paxinos G, Watson C (1998) The rat brain in stereotaxic coordinates. San Diego: Academic.

Peters Y, O’Donnell P, Carelli R (2005) Prefrontal cortical cell firing during maintenance, extinction, and reinstatement of goal-directed behavior for natural reward. Synapse 56:74-83.

Peterson J, Wolf M, White F (2000) Altered responsiveness of medial prefrontal cortex neurons to glutamate and dopamine after withdrawal from repeated amphetamine treatment. Synapse 36:342-344.
Porrino LJ, Lyons D (2000) Orbital and medial prefrontal cortex and psychostimulant abuse: studies in animal models. Cereb Cortex 10:326-333.

Post R, Kopanda R (1976) Cocaine, kindling, and psychosis. Am J Psychiatry 133:627-634.

Robbins T, Everitt B (2002) Limbic-striatal memory systems and drug addiction. Neurobiol Learn Mem 78:625-636.

Robinson T, Berridge K (1993) The neural basis of drug craving: an incentive-sensitization theory of addiction. Brain Res Brain Res Rev 18:247-291.

Robinson T, Berridge K (2002) Addiction. Annu Rev Psychol 54:25-53.

Robinson T, Kolb B (2004) Structural plasticity associated with exposure to drugs of abuse. Neuropharmacology 47 [Suppl 1]:33-46.

Robinson TE, Kolb B (1997) Persistent structural modifications in nucleus accumbens and prefrontal cortex neurons produced by previous experience with amphetamine. J Neurosci 17:8491-8497.

Rolls E (2004) Convergence of sensory systems in the orbitofrontal cortex in primates and brain design for emotion. Anat Rec A Discov Mol Cell Evol Biol 281:1212-1225.

Rolls E, Critchley H, Mason R, Wakeman E (1996) Orbitofrontal cortex neurons: role in olfactory and visual association learning. J Neurophysiol 75:1970-1981.

Schoenbaum G, Eichenbaum H (1995) Information coding in the rodent prefrontal cortex. II. Ensemble activity in orbitofrontal cortex. J Neurophysiol 74:751-762.

Schoenbaum G, Saddoris M, Ramus S, Shaham Y, Setlow B (2004) Cocaineexperienced rats exhibit learning deficits in a task sensitive to orbitofrontal cortex lesions. Eur J Neurosci 19:1997-2002.

Sorg B, Davidson D, Kalivas P, Prasad B (1997) Repeated daily cocaine alters subsequent cocaine-induced increase of extracellular dopamine in the medial prefrontal cortex. J Pharmacol Exp Ther 281:54-61.

Szumlinski K, Dehoff M, Kang S, Frys K, Lominac K, Klugmann M, Rohrer J, Griffin Wr, Toda S, Champtiaux N, Berry T, Tu J, Shealy S, During M, Middaugh L, Worley P, Kalivas P (2004) Homer proteins regulate sensitivity to cocaine. Neuron 43:401-413.

Thomas M, Malenka R (2003) Synaptic plasticity in the mesolimbic dopamine system. Philos Trans R Soc Lond B Biol Sci 358:815-819.

Trantham-Davidson H, Neely L, Lavin A, Seamans J (2004) Mechanisms underlying differential $D_{1}$ versus $D_{2}$ dopamine receptor regulation of inhibition in prefrontal cortex. J Neurosci 24:10652-10659.

Tremblay L, Schultz W (1999) Relative reward preference in primate orbitofrontal cortex. Nature 398:704-708.

Uylings H, Groenewegen H, Kolb B (2003) Do rats have a prefrontal cortex? Behav Brain Res 146:3-17.

Volkow N, Hitzemann R, Wang G, Fowler J, Wolf A, Dewey S, Handlesman L (1992) Long-term frontal brain metabolic changes in cocaine abusers. Synapse 11:184-190.

Volkow ND, Fowler JS (2000) Addiction, a disease of compulsion and drive: involvement of the orbitofrontal cortex. Cereb Cortex 10:318-325.

Wang G, Volkow N, Fowler J, Ding Y, Logan J, Gatley S, MacGregor R, Wolf A (1995) Comparison of two pet radioligands for imaging extrastriatal dopamine transporters in human brain. Life Sci 57:PL187-PL191.

Wise R, Bozarth M (1987) A psychomotor stimulant theory of addiction. Psychol Rev 94:469-492.

Wolf M (2002) Addiction: making the connection between behavioral changes and neuronal plasticity in specific pathways. Mol Interv 2:146-157.

Wolf ME (1998) The role of excitatory amino acids in behavioral sensitization to psychomotor stimulants. Prog Neurobiol 54:679-720. 